\title{
Large-amplitude pairing fluctuations in atomic nuclei
}

\author{
Nuria López Vaquero and J. Luis Egido* \\ Departamento de Física Teórica, Universidad Autónoma de Madrid, E-28049 Madrid, Spain
}

Tomás R. Rodríguez

Institut für Kernphysik, Technische Universität Darmstadt, Schlossgartenstrasse 2, D-64289 Darmstadt, Germany

(Received 5 August 2013; revised manuscript received 20 November 2013; published 10 December 2013)

\begin{abstract}
Pairing fluctuations are self-consistently incorporated on the same footing as the quadrupole deformations in present state-of-the-art calculations including particle-number and angular-momentum conservation as well as configuration mixing. The approach is complemented by the use of the finite-range density-dependent Gogny force which, with a unique source for the particle-hole and particle-particle interactions, guarantees a self-consistent interplay in both channels. We have applied our formalism to study the role of the pairing degree of freedom in the description of the most relevant observables like spectra, transition probabilities, separation energies, etc. We find that the inclusion of pairing fluctuations mostly affects the description of excited states, depending on the excitation energy and the angular momentum. E0 transition probabilities experience rather big changes while $E 2$ 's are less affected. Genuine pairing vibrations are thoroughly studied with the conclusion that deformations strongly inhibits their existence. These studies have been performed for a selection of nuclei: spherical, deformed, and with different degrees of collectivity.
\end{abstract}

DOI: 10.1103/PhysRevC.88.064311

PACS number(s): 23.20.Lv, 21.10.Re, 21.30.Fe, 21.60.Ev

\section{INTRODUCTION}

The self-consistent mean-field approach (MFA) with effective phenomenological interactions have succeeded in describing many bulk properties along the whole nuclear chart. This success is closely related to the incorporation of the spontaneous symmetry-breaking mechanism in the MFA, allowing thereby the inclusion of many correlations within a simple intrinsic product wave function (w.f.) [1].

Nonbulk observables require the use of beyond-mean-field theories (BMFTs). The description of nuclear spectra and transitions, for example, requires at least one more degree of sophistication, namely good quantum numbers. Therefore, the first step to improve the MFA is the restoration of the broken symmetries. This is usually done by projection techniques and it is obvious that we have to recover at least the main quantum numbers, i.e., the number of particles, the angular momentum, and the parity. This procedure is known as symmetry-conserving mean-field approximation (SCMFA) [2-4]. The self-consistent implementation of the projection is done in the variation after the projection (VAP) approach [5-7]; a poorer implementation is obtained in the projection after the variation (PAV) method. It is obvious that the former approach is much better because one varies w.f.'s with the right quantum numbers; the associated numerical difficulty is, however, much larger. In the case of the number of particles both approaches are in use but for the angular momentum and with effective forces only the PAV one is tractable.

In spite of the numerical complication the w.f.'s of the SCMFA behave in many aspects as mean-field ones because fluctuations around the most probable values are ignored. The inclusion of these fluctuations leads us directly to the next improvement, namely, the implementation of the configuration

*j.luis.egido@uam.es mixing technique. This is the so-called symmetry conserving configuration mixing (SCCM) [8-11] approach. This is performed within the generator coordinate method (GCM), which makes it possible to deal with collective degrees of freedom in a simple way. In this approach the GCM w.f. is expressed as a linear combination of basis states with different values of chosen collective coordinates. For the long-range part of the interaction one uses as collective coordinates the multipole expansion of the deformation operators, namely, quadrupole, octupole, hexadecupole, etc. For the short-range part the energy gap parameter seems to be the most appropriated collective coordinate. Because each degree of freedom increases considerably the CPU time one can consider explicitly only the most relevant ones. In practice only the quadrupole deformation is typically considered explicitly in axial or triaxial calculations. However, it is also well known that pairing correlations play a relevant role in the description of nuclear observables [12]. As a matter of fact, a proper description at the mean-field level is only achieved in the Hartree-FockBogoliubov (HFB) approach [13], which deals quadrupole and monopole degrees of freedom on the same footing. It seems therefore desirable to consider also the deviation of the mean-field values for both degrees of freedom in a BMFT.

Pairing fluctuations are an important degree of freedom in many nuclear phenomena and have been widely investigated [14-20].

In the gauge space associated with pairing, the HFB w.f. has two collective degrees of freedom: the pairing gap $\Delta$, which measures the amount of pairing correlations, i.e., the "deformation" [21] in the associated gauge space, and the angle $\varphi$, which indicates the orientation of the HFB state in this space. The HFB minimization determines the w.f. and thereby $\Delta$ while the gauge angle $\varphi$ does not play any role at the mean-field level. The degree of freedom associated with $\varphi$ has been exploited in the past [5]: Linear combinations of 
w.f.'s with different orientation in the gauge space provide a number-conserving w.f. Pairing vibrations-associated with w.f.'s with different pairing gaps_-around the average gap parameter of the energy minimum, however, have attracted little attention. As a matter of fact, they have been considered only either with very schematic interactions in the framework of the collective Hamiltonian [22-25], in microscopic model calculations [26,27], or in reduced configuration space [28]. In the approach closest to ours, by Meyer et al. [29], pairing vibrations were considered to study the stability of superdeformed shapes in ${ }^{194} \mathrm{Hg}$ in the framework of the GCM with the Skyrme force. In these calculations, however, neither particle-number nor angular-momentum projection were considered. These projections are very important not only to have good quantum numbers but also because the Hamiltonian and norm overlaps are quite different, changing completely the dynamic of the system.

Recently, we published a Letter [30] that simultaneously considers the quadrupole and the pairing degrees of freedom in the SCCM framework with simultaneous particle-number and angular-momentum projections. This first study was limited to analyzing the impact of the pairing fluctuations on the spectrum of the nucleus ${ }^{54} \mathrm{Cr}$. The purpose of this paper is to extend the analysis to other observables like transition probabilities, separation energies, pairing vibrations, etc., as well as to explore different parts of the chart of nuclides.

The paper is organized as follows. In Sec. II the basic theoretical formulation is provided. In Sec. III the practical implementation of the theory is shown. In Sec. IV the potential energy surfaces are discussed for the one- and two-dimensional case. The norm overlaps are considered in Sec. V and the energy spectra for several nuclei are displayed in Sec. VI. The collective w.f.'s are discussed in Sec. VII. The existence of genuine pairing vibration is tackled in Sec. VIII and the particle-number distribution of non-particle-number projected w.f.'s is discussed in Sec. IX. Finally, some miscellaneous calculations are discussed in Sec. X.

\section{THEORETICAL FRAMEWORK}

The starting point of any BMFT is the generation of the HFB basis states in which the GCM w.f. can be expanded. According to the different coordinates to be considered in the calculations, the HFB w.f.'s $|\phi\rangle$ have to be calculated in each point of the grid subtended by those coordinates. This is best achieved within the constrained HFB approach in conjunction with the conjugate gradient method [31]. In the case of the multipole coordinates the operators to be constrained are the corresponding operators. For the pairing coordinate and for finite-range interactions it has turned out [30] that the more convenient operator to be constrained is $\Delta \hat{N}^{2}=(\hat{N}-\langle\phi|| \hat{N} \mid \phi\rangle)^{2}, \hat{N}$ being the number of particles, protons, or neutrons. For state-independent pairing interactions, this operator is closely connected with the energy pairing gap $\Delta[30]$.

In this work we restrict ourselves to two coordinates, the axially symmetric quadrupole deformation, $\hat{Q}_{20}$ and the pairing correlations. The way to proceed to implement the corresponding fluctuations is as follows. One generates a set of intrinsic HFB w.f.'s $|\phi\rangle$ with given quadrupole deformation $q$ and "pairing deformation" $\delta$ by solving the variational equation

$$
\delta E^{\prime}[\phi(q, \delta)]=0,
$$

with

$$
E^{\prime}=\frac{\langle\Phi|\hat{H}| \Phi\rangle}{\langle\Phi \mid \Phi\rangle}-\lambda_{q}\left\langle\phi\left|\hat{Q}_{20}\right| \phi\right\rangle-\lambda_{\delta}\left\langle\phi\left|(\Delta \hat{N})^{2}\right| \phi\right\rangle^{1 / 2},
$$

and the Lagrange multipliers $\lambda_{q}$ and $\lambda_{\delta}$ being determined by the constraints

$$
\left\langle\phi\left|\hat{Q}_{20}\right| \phi\right\rangle=q, \quad\left\langle\phi\left|(\Delta \hat{N})^{2}\right| \phi\right\rangle^{1 / 2}=\delta .
$$

It remains to specify the meaning of the w.f. $|\Phi\rangle$. One has to distinguish several cases: In the more elaborated approach $|\Phi\rangle=\hat{P}^{N}, \ldots, \hat{P}^{I}|\phi\rangle$, with $\hat{P}$ an operator that restores the symmetry (symmetries) broken by the intrinsic w.f. $|\phi\rangle$. In this case we are solving the self-consistent SCMFA, i.e., in the VAP approach. In our case we are interested in restoring the rotational invariance and the number of particles symmetry; i.e., we should take $|\Phi\rangle=\hat{P}^{N} \hat{P}^{I}|\phi\rangle$, where $\hat{P}^{N}$ performs the particle-number projection (PNP) and $\hat{P}^{I}$ the angular-momentum projection (AMP). This is the most general SCMFA and it is very CPU time consuming. However, taking into account that, in general, the semiclassical approximation of using Lagrange parameters is a very good approximation for strong collective symmetry breaking as in the case of the angular momentum - and less appropriated for non very collective symmetry like the particle number-we shall consider $|\Phi\rangle=\hat{P}^{N}|\phi\rangle$. That means, in this case, only the particle-number restoration is considered in the VAP approach, whereas the AMP is performed after the variation has taken place, i.e., in the PAV approach. We call this procedure PNVAP approach. This method although more involved provides a much better description of the pairing correlations in the intrinsic w.f. [7]. In the simplest case $|\Phi\rangle \equiv|\phi\rangle$ and, therefore, we are solving the plain HFB equations. Notice that in this case we have to add, to the right-hand side of Eq. (2), the term $-\lambda_{N} \hat{N}$ to keep the right number of particles; on the average, $\lambda_{N}$ is fixed by the constraint $\langle\phi|\hat{N}| \phi\rangle=N$. In this case both the PNP and the AMP are performed in the PAV approach.

Once we have generated the basis states we can proceed with the configuration mixing calculation. This is carried out within the GCM taking linear combinations of the w.f.'s obtained in the first step after performing projections on the required symmetries,

$$
\left|\Psi^{b, \sigma}\right\rangle=\int f^{b, \sigma}(q, \delta) \hat{P}^{\mathrm{B}}|\phi(q, \delta)\rangle d q d \delta,
$$

where $b$ denotes the set of quantum numbers onto which $\hat{P}^{\mathrm{B}}$ projects, in the most general case $b=I, N, Z{ }^{1}$ Then, the variational principle applied to the weights $f^{b, \sigma}(q, \delta)$ gives

\footnotetext{
${ }^{1}$ To simplify the notations in this paper we do not distinguish between protons and neutrons; thus, for example, when we write $\hat{P}^{B}=\hat{P}^{N} \hat{P}^{I}$, we mean $\hat{P}^{\mathrm{B}}=\hat{P}^{Z} \hat{P}^{N} \hat{P}^{I}$, with $Z$ and $N$ the proton and neutron numbers, respectively.
} 
the generalized eigenvalue problem, the Hill-Wheeler (HW) equation,

$$
\int\left[\mathcal{H}^{b}\left(q \delta, q^{\prime} \delta^{\prime}\right)-E^{b, \sigma} \mathcal{N}^{b}\left(q \delta, q^{\prime} \delta^{\prime}\right)\right] f^{b, \sigma}\left(q^{\prime} \delta^{\prime}\right) d q^{\prime} d \delta^{\prime}=0,
$$

with $\mathcal{H}^{b}$ and $\mathcal{N}^{b}$ the Hamiltonian and norm overlaps, respectively, and $\sigma=1,2, \ldots$, numbers the ground and excited states with quantum numbers $b, E^{b, \sigma}$ is the energy of the corresponding state, and $\sigma=1$ being the yrast state. See Refs. [10,11] for a detailed description of the solution of the HW equation. The collective w.f.'s are provided by [32]

$$
g^{b, \sigma}(q \delta)=\sum_{q^{\prime} \delta^{\prime}} \mathcal{N}^{b^{1 / 2}}\left(q \delta, q^{\prime} \delta^{\prime}\right) f^{b, \sigma}\left(q^{\prime} \delta^{\prime}\right),
$$

which are orthogonal and satisfy $\sum_{q \delta}\left|g(q \delta)^{b, \sigma}\right|^{2}=1$; the weights $f^{b, \sigma}(q \delta)$, however, do not satisfy these properties.

In the case where in Eq. (4) the operator $\hat{P}^{B}=\hat{P}^{I}$, i.e., without PNP, because the constraint on the particle number is done at the HFB level, nothing guarantees that $\hat{P}^{I}|\phi(q, \delta)\rangle$ or $\left|\Psi^{I, \sigma}\right\rangle$ have the right values for the number of protons and neutrons. To correct for this deficiency the usual cranking recipe [32] of minimizing $\hat{H}^{\prime}=H-\lambda \hat{N}$ instead of $H$ is used. This amounts to substituting $\mathcal{H}$ with $\mathcal{H}^{\prime}$ in Eq. (5), with $\mathcal{H}^{\prime}$ given by

$$
\left(\mathcal{H}^{\prime}\right)^{I}\left(q \delta, q^{\prime} \delta^{\prime}\right)=\mathcal{H}^{I}\left(q \delta, q^{\prime} \delta^{\prime}\right)-\lambda \Delta N^{I}\left(q \delta, q^{\prime} \delta^{\prime}\right),
$$

where the parameter $\lambda$ is an average of the Lagrange parameters $\lambda_{N}$ of the HFB equations in the different $(q \delta)$ points and $\Delta N^{I}\left(q \delta, q^{\prime} \delta^{\prime}\right)=\left\langle\phi(q \delta)\left|(\hat{N}-N) P^{I}\right| \phi\left(q^{\prime} \delta^{\prime}\right)\right\rangle[33]$.

\section{DETAILS OF THE CALCULATIONS}

We use in our numerical application the finite-range density-dependent Gogny force with the D1S parametrization [34]. A detailed description of the evaluation of the different terms of the Hamiltonian in the HFB and in the PN-VAP can be found in Ref. [7] and in the AMP and GCM in Refs. $[10,11]$. The density-dependent term of the interaction has been scrutinized in the last decade [7,35-37]; we therefore provide a short discussion of our approach to this term in the Appendix. To have a tractable problem we limit our configuration space to eight spherical [38] oscillator shells. Though we concentrate on small nuclei, this configuration space, in some cases, might be too small to provide quantitative results. As already mentioned, we limit ourselves also to axially symmetric deformations.

We present three different methods based on different types of projections explained above, namely, HFB + AMP, HFB + PNAMP, and PN-VAP + PNAMP. In the first one (HFB + AMP) PNP is completely ignored; i.e., the intrinsic w.f., $|\phi(q, \delta)\rangle$, is determined in the HFB approach and afterwards AMP is performed in the configuration mixing procedure, in this case $P^{B}=P^{I}$. In the second one, (HFB + PNAMP) $|\phi(q, \delta)\rangle$ is determined in the HFB approach but then PNP and AMP are performed in the configuration mixing method; i.e, now $P^{B}=P^{I} P^{N}$. In the last one (PN-VAP + PNAMP), $|\phi(q, \delta)\rangle$ is determined in the PN-VAP method and afterwards the PNP and AMP are performed in the configuration mixing mechanism. Furthermore, to investigate the effect of the pairing fluctuations we present results in one dimension (1D), i.e., only with the quadrupole moment as coordinate generator and in two dimensions (2D) with the pairing energy and the quadrupole moment as generator coordinates.

In principle, the calculations should be 3D with coordinates $\left(q, \delta_{Z}, \delta_{N}\right)$ with separate constraints for neutrons and protons,

$$
\left\langle\phi\left|(\Delta \hat{N})^{2}\right| \phi\right\rangle^{1 / 2}=\delta_{N}, \quad\left\langle\phi\left|(\Delta \hat{Z})^{2}\right| \phi\right\rangle^{1 / 2}=\delta_{Z} ;
$$

unfortunately, with three constraints the problem becomes computationally very demanding. What we have done is substitute the above constraints with a single one on $\delta$, the Lagrange multiplier $\delta$ being defined by

$$
\left\langle\phi\left|(\Delta \hat{N})^{2}\right| \phi\right\rangle^{1 / 2}+\left\langle\phi\left|(\Delta \hat{Z})^{2}\right| \phi\right\rangle^{1 / 2}=\delta .
$$

To check the quality of our approximation we have performed three kinds of calculations. In the first one we have constrained only $\delta_{N}=\left\langle\phi\left|(\Delta \hat{N})^{2}\right| \phi\right\rangle^{1 / 2}$; the variational principle guarantees that in this case $\left\langle\phi\left|(\Delta \hat{Z})^{2}\right| \phi\right\rangle^{1 / 2}$ will self-consistently take the optimal value as to minimize the energy. In the second one we constrain only on $\delta_{Z}=\left\langle\phi\left|(\Delta \hat{Z})^{2}\right| \phi\right\rangle^{1 / 2}$, whereas $\left\langle\phi\left|(\Delta \hat{N})^{2}\right| \phi\right\rangle^{1 / 2}$ is allowed to vary freely. In the third one we constraint on $\delta=\left\langle\phi\left|(\Delta \hat{N})^{2}\right| \phi\right\rangle^{1 / 2}+\left\langle\phi\left|(\Delta \hat{Z})^{2}\right| \phi\right\rangle^{1 / 2}$. We have performed calculations in the PN-VAP + PNAMP approach for the nucleus ${ }^{50} \mathrm{Ca}$; we have chosen this nucleus because it is magic in protons and in this case it would be clearer to decide on the quality of the approaches. The ground-state energies are $-428.991 \mathrm{MeV}$ (constraint on $\delta_{Z}$ ),$-428.962 \mathrm{MeV}$ (constraint on $\delta_{N}$ ), and $-429.037 \mathrm{MeV}$ (constraint on $\delta$ ). Though the lowest energy is obtained in the case where we constrain on $\delta$ we do not find significant differences in the three calculations. Concerning the excitation energies and other relevant observables we present in Table I, the excitation energies of the three low-lying states for angular momentum $0^{+}$and $2^{+}$and the proton and neutron pairing energies in the three mentioned calculations, see [7] for the definition of the pairing energy. A close look at these results shows again that there are not remarkable differences. Besides, the same type of calculations for the nucleus ${ }^{32} \mathrm{Mg}$ reconfirm these findings. In conclusion, the constraint in $\delta$ is appropriate because one

\begin{tabular}{|c|c|c|c|c|c|c|c|c|c|}
\hline & \multicolumn{3}{|c|}{$\delta_{Z}$} & \multicolumn{3}{|c|}{$\delta_{N}$} & \multicolumn{3}{|c|}{$\delta$} \\
\hline & $E^{*}$ & $E_{\text {pair }}^{Z}$ & $E_{\text {pair }}^{N}$ & $E^{*}$ & $E_{\text {pair }}^{Z}$ & $E_{\text {pair }}^{N}$ & $E^{*}$ & $E_{\text {pair }}^{Z}$ & $E_{\text {pair }}^{N}$ \\
\hline $0_{1}^{+}$ & 0.00 & -4.1 & -5.9 & 0.00 & -4.2 & -6.2 & 0.00 & -4.0 & -5.7 \\
\hline $0_{2}^{+}$ & 4.58 & -5.0 & -5.9 & 4.80 & -4.5 & -6.0 & 4.65 & -4.7 & -5.7 \\
\hline $0_{3}^{+}$ & 7.80 & -5.1 & -4.7 & 6.76 & -4.7 & -4.3 & 7.17 & -4.1 & -3.7 \\
\hline $2_{1}^{+}$ & 2.60 & -4.0 & -4.0 & 2.11 & -4.0 & -3.1 & 2.21 & -3.5 & -3.2 \\
\hline $2_{2}^{+}$ & 5.75 & -4.7 & -5.2 & 5.31 & -4.5 & -3.3 & 5.59 & -4.2 & -4.2 \\
\hline $2_{3}^{+}$ & 6.05 & -4.2 & -4.0 & 5.88 & -4.6 & -4.9 & 5.90 & -3.7 & -4.0 \\
\hline
\end{tabular}
does not look for specific proton or neutron observables.

TABLE I. Excitation energy $E^{*}$, proton (neutron) pairing energy $E_{\text {pair }}^{Z(N)}$ for the three lowest $0^{+}$and $2^{+}$states of the HW equation in the PN-VAP + PNAMP constraining on $\delta_{Z}, \delta_{N}$, or $\delta$. 


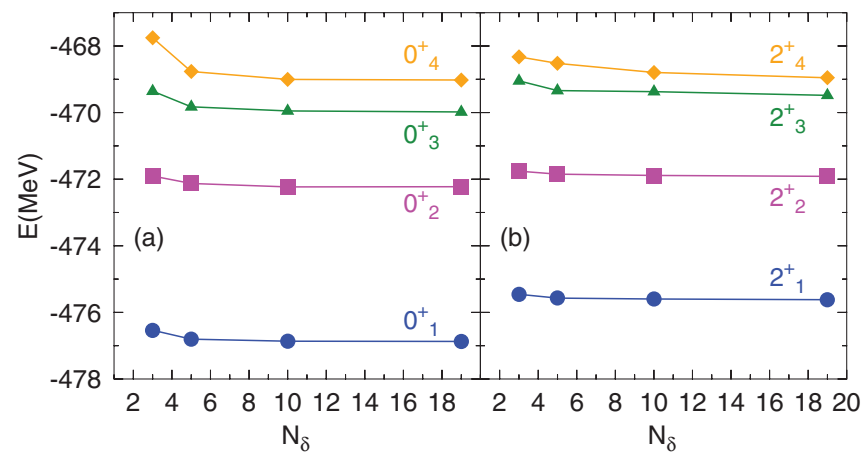

FIG. 1. (Color online) Energies of the lowest $0^{+}$and $2^{+}$states as a function of the number of $\delta$ points $(3,5,10$, or 19) used in the calculations in the PN-VAP + PNAMP approach for ${ }^{54} \mathrm{Cr}$. The lines connecting the points are to guide the eye.

For the $q$ coordinate we take an interval $-220 \mathrm{fm}^{2}$ up to $+400 \mathrm{fm}^{2}$ with $\Delta q=20 \mathrm{fm}^{2}$, for the pairing coordinate $\delta$ from 0 to 4.5 with a 0.5 interval. The chosen $\delta$ interval covers [30] a pairing energy range from 0.0 up to $\sim 50 \mathrm{MeV}$, to compare with typical values of a few MeV's in the nucleus we analyze. We have, therefore, a grid with 32 points for the unidimensional calculations ( $q$ coordinate) and with 320 for the $2 \mathrm{D}(q, \delta)$ ones.

The size of the grid and the size interval in $q$ has been discussed in the past. Because we are introducing a new coordinate, we are interested in knowing the suitable size of the basis concerned to the $\delta$ degree of freedom. In Fig. 1 we show solutions of Eq. (5) calculated in the PN-VAP + PNAMP approach in $2 \mathrm{D}$ for the nucleus ${ }^{54} \mathrm{Cr}$ for different number, $N_{\delta}$, of $\delta$ values $(3,5,10$, or 19$)$ in the interval 0 to 4.5 . We can conclude comparing these results that ten values of $\delta$ for each deformation is a good election for our calculations to guarantee a good energy convergence. Furthermore, the tails of the collective w.f.'s go to zero inside the interval border.

An inherent difficulty to the solution of the HW equation [Eq. (5)] is the fact that the basis states $\phi(q \delta)$ are not orthogonal. To overcome this problem [32] one works in the natural basis where the linear-dependent states do have zero or very small norm. The natural states with a norm smaller than a given cutoff are omitted in the final diagonalization of the HW equation. The determination of the cutoff is not always easy and one ends up studying the convergence of the energy and the corresponding w.f. as a function of the number of the natural basis states, $N_{\text {n.s. }}$, taken in the calculations. The signal for the convergence is the presence of a region where the energies are nearly constant, i.e., the so-called plateaus [33]. We now study the convergence of the four lowest $I=0^{+}$states in the different approximations. In Figs. 2 and 3 we show the convergence for the nuclei ${ }^{24} \mathrm{Mg}$ and ${ }^{32} \mathrm{Mg}$, respectively. We represent the energy, for the $1 \mathrm{D}$ and the $2 \mathrm{D}$ cases, versus $N_{\text {n.s. }}$. The final choice for $N_{\text {n.s. }}$ is a value around which we observe a wide plateau for all the levels of interest and the collective w.f.'s do not change. This value must be kept constant for a given angular momentum to guarantee the orthogonality of the corresponding w.f.'s.

In the actual calculations the natural states are ordered by decreasing norm eigenvalues and the general behavior that

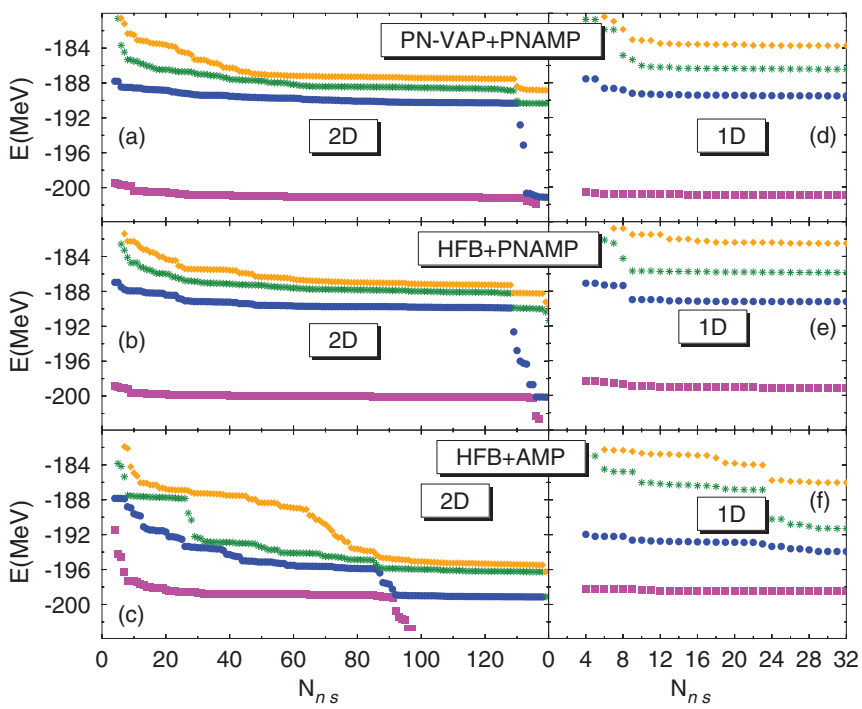

FIG. 2. (Color online) Energies of the four lowest $0^{+}$states of the nucleus ${ }^{24} \mathrm{Mg}$. Left (right) panels correspond to the 2D (1D) calculation both of them have been performed with the three different approaches.

one expects for the convergence as the number of natural basis states increases is the following. As long as the norm eigenvalues are relatively large, one expects a lowering of the energy with increasing number of natural states because the Hilbert space gets larger. We also expect faster convergence for low-lying energy eigenvalues. As the norm eigenvalues get smaller their contribution to the energy becomes very small and a kind of plateau is expected. However, because the linear-dependent states correspond to very small norm eigenvalues one must be aware because by increasing the number of natural states one definitively reaches a point with such small norms (close to zero) that the energy tends to very large negative values. This is the general behavior and, depending on the linear dependence and other aspects, one can have some variations, as can be seen in the depicted cases.

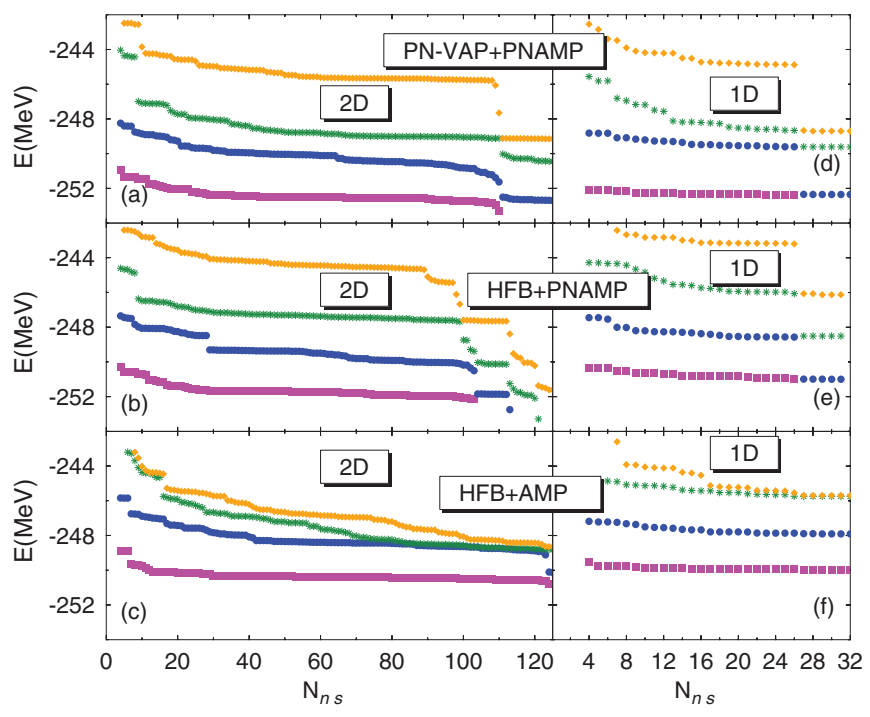

FIG. 3. (Color online) The same plot as Fig. 2, but for the ${ }^{32} \mathrm{Mg}$. 
The general behavior is clearly illustrated in Fig. 2(a). For small number of natural states the lowering of the energy is considerable; the ground state and the first excited state are converging very quickly. Taking into account 60 natural states, we obtain a rather good convergence for all four energy eigenstates. The consideration of additional natural states, up to about 120 , provides very small changes in the energy. Finally, by $N_{\text {n.s. }} \approx 130$ the norm of these states gets so small that its contribution to the energy is spurious. One observes that one energy eigenvalue decreases more and more as new natural states are considered. This spurious state crosses the physical ones (one should not get confused by the colors provided by the plotting code which assigns a given color to the lowest energy, magenta in this case, blue to the second and so on) and becomes more and more negative. By $N_{\text {n.s. }} \approx 140$ this spurious state provides not only huge energy but also huge values for any other observable one tries to calculate. For very large linear dependence on the basis, further spurious states can be obtained for even larger $N_{\text {n.s. }}$. In the opposite situation of small basis with small or no linear dependence, as in the 1D case, either nonspurious states appear, as in Figs. 2(d), 2(e), and 2(f) or the spurious state appears as a degenerated energy eigenvalue that never gets very large; see Fig. 3(f).

In the 2D case we find very wide plateaus in the PN-VAP + PNAMP and in the HFB + PNAMP cases, confirming a very good convergence, and not very good plateaus for the HFB + AMP approach, indicating a poorer convergence. These plots show that in the absence of PNP there is more mixing and larger linear dependence. We will see later that this peculiarity appears also for other properties. Concerning the 1D case the situation is similar to the 2D one. It appears to be less dramatic but this is attributable to the fact that the linear dependence is smaller because of the smaller size of the basis. For angular momentum larger than zero the situation is very similar to the one depicted here. The behavior of the HFB + PNAMP approach is not specific to the nucleus ${ }^{24} \mathrm{Mg}$, as can be seen in Fig. 3, where we display the convergence for the nucleus ${ }^{32} \mathrm{Mg}$. We find that the conclusions drawn for ${ }^{24} \mathrm{Mg}$ do also apply for ${ }^{32} \mathrm{Mg}$.

\section{POTENCIAL ENERGY SURFACES}

In this and in the next sections we discuss several relevant properties for a variety of nuclei: deformed, spherical, collective, etc.

\section{A. One-dimensional case}

A useful quantity for the interpretation of observables is the diagonal of the Hamiltonian overlap entering into the HW matrix, Eq. (5), as a function of the generator coordinate(s). In the $1 \mathrm{D}$ case we are concerned with $\left\langle\phi(q)\left|H P^{B}\right| \phi(q)\right\rangle /\left\langle\phi(q)\left|P^{B}\right| \phi(q)\right\rangle$, where $P^{B} \equiv P^{I}$ or $P^{B} \equiv$ $P^{I} P^{N}$, depending on the approach. In Fig. 4 (left panels), we present the potential energy curves for the nuclei ${ }^{52} \mathrm{Ti},{ }^{24} \mathrm{Mg}$, and ${ }^{32} \mathrm{Mg}$ for $I=0 \hbar$ versus the quadrupole moment.

In the PN-VAP + PNAMP approach the nucleus ${ }^{52} \mathrm{Ti}$ presents coexistent prolate and oblate shapes and a prolate superdeformed shoulder. In the ${ }^{24} \mathrm{Mg}$ case we obtain a strongly

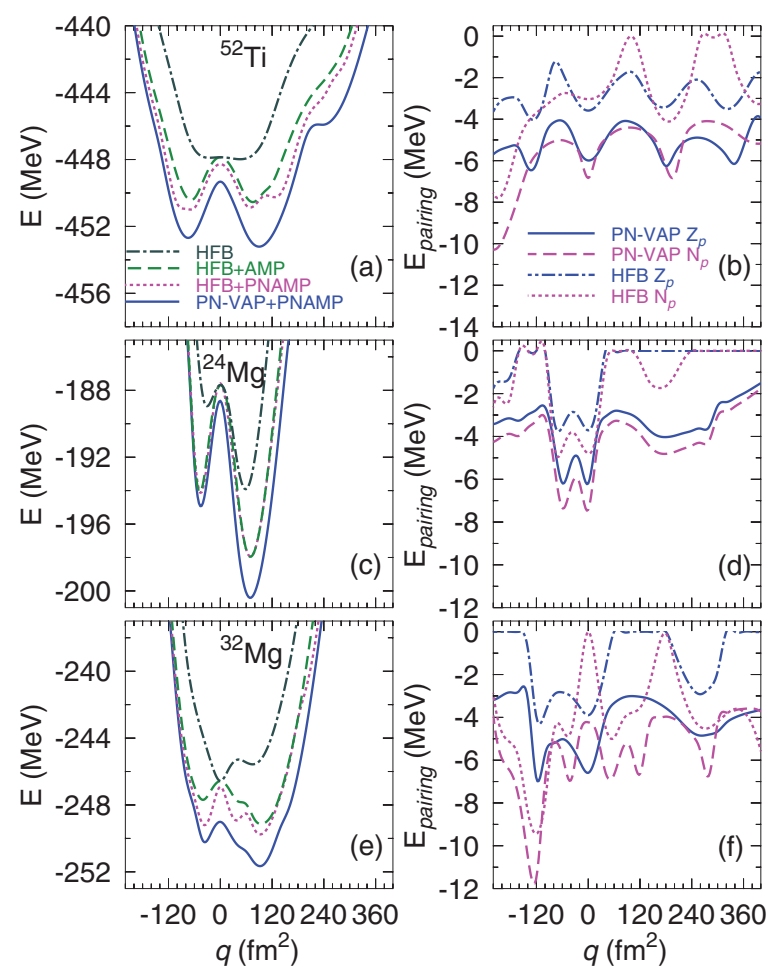

FIG. 4. (Color online) (Left panels) One-dimensional potential energy curves for the nuclei ${ }^{52} \mathrm{Ti},{ }^{24} \mathrm{Mg}$, and ${ }^{32} \mathrm{Mg}$ in the HFB and in several approaches for $I=0^{+}$. (Right panels) Proton and neutron pairing energies of the intrinsic w.f.'s in the HFB and PN-VAP for the same nuclei.

prolate deformed minimum and about $5 \mathrm{MeV}$ above an oblate one. For ${ }^{32} \mathrm{Mg}$ we obtain a prolate deformed minimum and an oblate pocket about $2 \mathrm{MeV}$ above. These three nuclei correspond to scenarios representative of the different cases one may find. The HFB + PNAMP and HFB + AMP curves display more or less similar shapes to the PN-VAP + PNAMP ones but lie a few $\mathrm{MeV}$ above. In all cases, the energy gain of the PN-VAP + PNAMP approach is the largest one. In the right panels of the same figure the pairing energies of the intrinsic w.f.'s in the HFB and in the PN-VAP approach are displayed. In the ${ }^{52} \mathrm{Ti}$ nucleus we find an oscillatory behavior as a function of the deformation. In the HFB approach we observe a collapse of the neutron pairing correlations at the $q$ values corresponding to the prolate minimum and the superdeformed pocket. In the PN-VAP approach we do not observe any collapse but we obtain larger absolute values. The same trend is observed in the magnesium isotopes: pairing collapse at the minima for the HFB approach and much larger values for the PN-VAP one. The fact that we do not obtain the same correlations for the $N=Z{ }^{24} \mathrm{Mg}$ nucleus has to do with the Coulomb anti-pairing (CouAP) effect (CouAP) [39].

\section{B. Two-dimensional case}

We study now the dependence of the potential energy of these nuclei with respect to the two collective degrees of freedom $(q, \delta)$. In Fig. 5, we present contour lines of the potential energy of ${ }^{52} \mathrm{Ti}$ as function of the constrained 

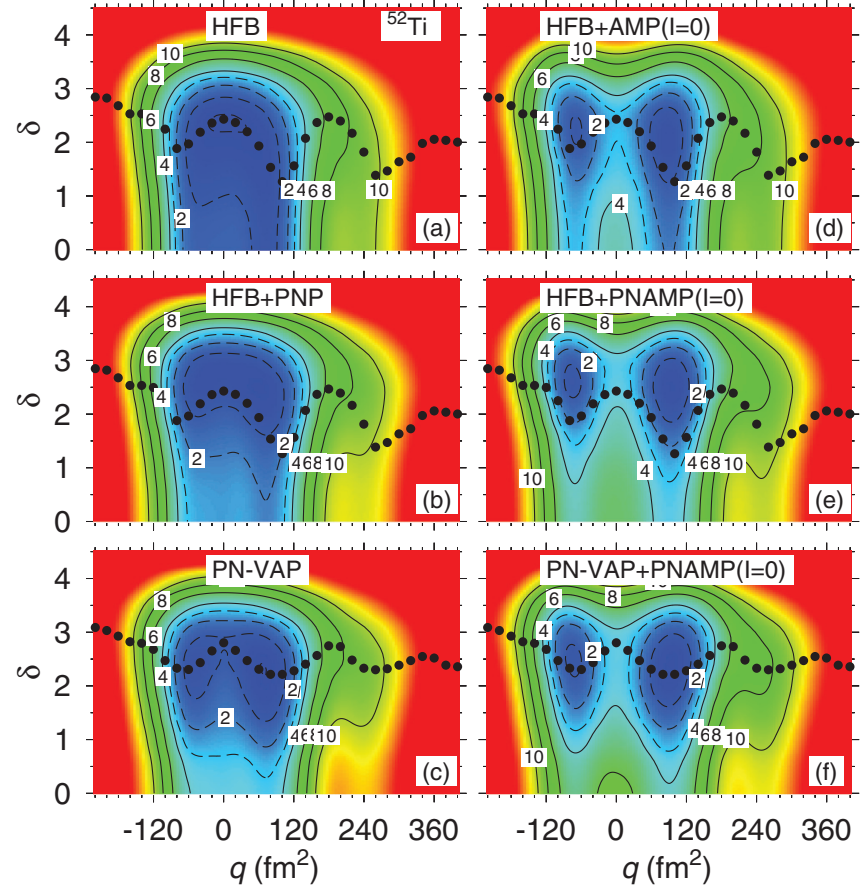

FIG. 5. (Color online) Potential energy contour plots for ${ }^{52} \mathrm{Ti}$ in the $(\delta, q)$ plane in different approaches. The dashed lines show equipotential lines from 0 to $3 \mathrm{MeV}$ in steps of $1 \mathrm{MeV}$. The continuous lines are contours from 4 to $10 \mathrm{MeV}$ in steps of $2 \mathrm{MeV}$. In each panel the energy origin has been chosen independently and the energy minimum has been set to zero. The bullets in each panel represent the $\delta$ values of the self-consistent solution (HFB or PN-VAP) extracted from the 1D ( $q$-constrained) approach and are displayed as a discussion guide. Because all HFB based approaches have the same intrinsic w.f., all of them have the same bullet pattern. The same applies to all PN-VAP-based approaches.

parameters $(q, \delta)$ in different approximations. The bullets represent the $\delta$ values of the self-consistent solutions (HFB or PN-VAP, i.e., without AMP) of the 1D ( $q$-constrained) approach. They must be orthogonal to the equipotentials curves in the corresponding approach. The 1D plots of Fig. 4 can be used as a guide in the interpretation of the $2 \mathrm{D}$ plots.

The relationship between the parameter $\delta$ and the pairing energy is rather independent of the $q$ value; see Fig. 2 of Ref. [30]. To get a feeling for the nucleus ${ }^{52} \mathrm{Ti}$ and for $q=100 \mathrm{fm}^{2}$ in the VAP + PNAMP approach and for $I=0$, we provide the pairing energy (in parentheses and in $\mathrm{MeV}$ ) corresponding to the preceding $\delta$ values: $0.0(0.00), 0.5(-0.52)$, $1.0(-2.11), \quad 1.5(-4.74), \quad 2.0(-8.19), \quad 2.5(-12.53)$, $3.0(-18.33), 3.5(-26.17), 4.0(-36.71)$, and 4.5(-50.26). We thus see that the $\delta$ range covers a wide energy interval.

In Fig. 5(a) we display the pure HFB case. Here we find a region delimited from $q=-60 \mathrm{fm}^{2}$ to $q=100 \mathrm{fm}^{2}$ in the $X$ axis and from $\delta=0$ to $\delta=2.5$ in the ordinate, where the potential is soft in both directions. That means, for a given value of $q$ (or $\delta$ ) one does not gain much energy (just around $1 \mathrm{MeV}$ ) by increasing the $\delta$ coordinate (or $q$ ). However, for the same $q$ interval, but $\delta$ between 2.5 and 4 , it takes a considerable amount of energy to increase the pairing correlations of the system. For higher values of $\delta$, the pairing energy gain is huge and the total energy is up to $20 \mathrm{MeV}$ larger. An analogous conclusion is obtained for the region $-140 \mathrm{fm}^{2}$ $<q<-60 \mathrm{fm}^{2}$ and $120 \mathrm{fm}^{2}<q<240 \mathrm{fm}^{2}$, the potential becomes stiff and to deform the nucleus to that value requires a large amount of energy. This structure is consistent with the 1D plot shown in the top left panel of Fig. 4.

Next, in panel (b) we show the effect of particle-number PAV; i.e., one takes the HFB w.f.'s used to generate panel (a) and calculates the PNP energy. One obtains again rather flat minima but displaced to $\delta=2.5$. The energy lowering of the absolute minimum is $1.37 \mathrm{MeV}$. In panel (c) we also represent the effect of the PNP but in this case, the projection is performed before the variation; therefore, in that approximation we obtain the energy with PN-VAP intrinsic w.f.'s. This plot looks like the previous one and the two trends mentioned before are present here too: The equipotentials are shifted towards large values of $\delta$ and the minimum is deeper, being now even lower, $1.17 \mathrm{MeV}$ below the PAV absolute minimum. One now observes two minima, one prolate at $\left(q=60 \mathrm{fm}^{2}, \delta=2.5\right)$ and one oblate at $\left(q=-40 \mathrm{fm}^{2}\right.$, $\delta=2.5$ ). The PN-VAP approach is the proper way to perform the variation because one minimizes the energy calculated with the right number of particles. One has to keep in mind that, even though the PNP brings the energy minimum of the HFB solution closer to the VAP one, there are other observables whose values do not coincide with the self-consistent ones provided by the VAP approach.

Now, the AMP $(I=0 \hbar)$ is performed for the approaches of the left panels and presented in the corresponding right panels. We start with the HFB + AMP case [panel (d) here, as mentioned above; see Eq. (7)]; in each $(\delta, q)$ point we have performed the particle-number energy correction owing to the fact that the AMP w.f. does not provide, on the average, the right particle number. As seen in Fig. 4 the AMP increases considerably the depth of the potentials and the $q$ values of the minima. They move to larger $q$ values, $-80 \mathrm{fm}^{2}$ for the oblate minimum and $80 \mathrm{fm}^{2}$ for the prolate one. In the $\mathrm{HFB}+\mathrm{PNAMP}$ [panel (e)] or in the PN-VAP + PNAMP [panel (f)] the effect of the AMP is also to widen the equipotentials and to deepen the minima, the prolate being shifted towards larger value, $100 \mathrm{fm}^{2}$, and the oblate one to $-80 \mathrm{fm}^{2}$. An interesting point is that in the $2 \mathrm{D}$ plot we find that the minima of the energy in the HFB + AMP approach correspond to pairing energies of $\delta \approx 2.0$. We find that this is not the case in the PNP cases where the minima correspond to $\delta \approx 2.5$. The energy difference corresponding to the different $\delta$ values amounts to a difference of pairing energies of a few MeV's [30]. The equipotential surfaces of panels (e) and (f) look very similar, though in detail they are different; cf. Fig. 4. The fact that the minima of the HFB + AMP approach lie at a weak pairing region will have important consequences because the masses associated with the dynamics of the system, i.e., the solution of the HW equation, will be much larger than the ones associated with the PN projected approaches, providing a more compressed spectrum. The energy gain of the absolute minimum in the PN-VAP + PNAMP approach with respect to the HFB (PN-VAP) is $4.53 \mathrm{MeV}(2.711 \mathrm{MeV})$.

Let us now turn to the magnesium isotopes. In Fig. 6 we display the potential energy surfaces for ${ }^{24} \mathrm{Mg}$ and ${ }^{32} \mathrm{Mg}$ 

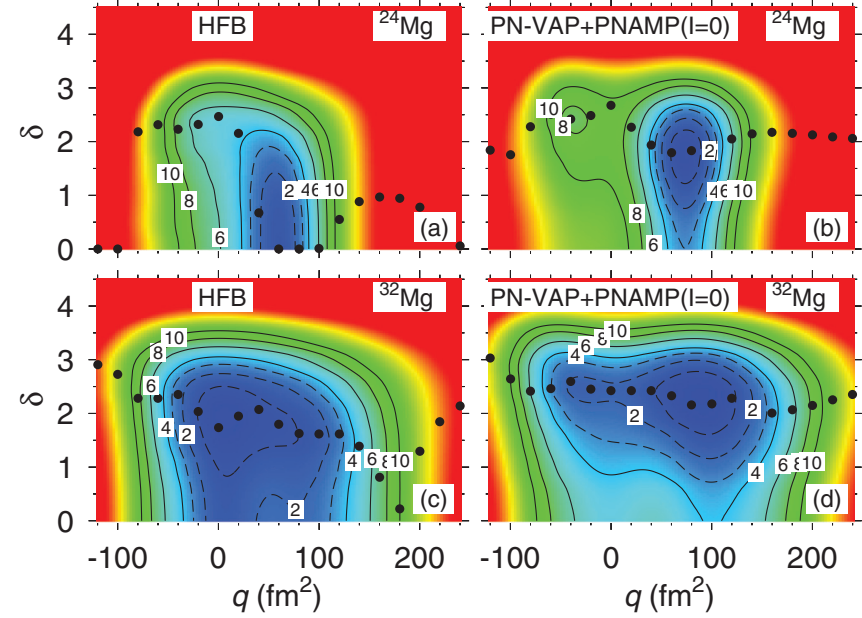

FIG. 6. (Color online) (Left panels) Potential energy surfaces for the nuclei ${ }^{24} \mathrm{Mg}$ and ${ }^{32} \mathrm{Mg}$ in the HFB approach. (Right panels) Potential energy surfaces for the nuclei ${ }^{24} \mathrm{Mg}$ and ${ }^{32} \mathrm{Mg}$ in the PN-VAP + PNAMP approach for $I=0$. In both cases the contours follow the same interval as in Fig. 5.

just in the HFB and in the PN-VAP + PNAMP approach for $I=0 \hbar$. In the first row we find that ${ }^{24} \mathrm{Mg}$ displays a stiff potential in the HFB approach; it presents a structure of a deep prolate minimum $\left(q \approx 80 \mathrm{fm}^{2}\right)$ with $\delta=0$ and a few $\mathrm{MeV}$ higher an oblate one $\left(q \approx-30 \mathrm{fm}^{2}\right)$. We observe that this nucleus is more steep towards larger pairing correlations than that seen in ${ }^{52} \mathrm{Ti}$. In the PN-VAP + PNAMP case the prolate minimum shifts to $q \approx 100 \mathrm{fm}^{2}$ and $\delta \approx 2.0$ and the oblate one to $q \approx-40 \mathrm{fm}^{2}$ and $\delta \approx 2.5$, the energy becoming even stiffer around the prolate minimum. In the second row we display ${ }^{32} \mathrm{Mg}$; in the HFB approach the energy minimum has a spherical shape and $\delta \approx 1.6$. About $2 \mathrm{MeV}$ higher there is a prolate shoulder with $q \approx 80 \mathrm{fm}^{2}$ and $\delta \approx 1.5$. In the PN-VAP + PNAMP approach, right panel, we observe two deformed minima, the deepest one at $q \approx 90 \mathrm{fm}^{2}$ and $\delta \approx 2.1$ and the oblate one at $q \approx-40 \mathrm{fm}^{2}$ and $\delta \approx 2.5$, about $2 \mathrm{MeV}$ higher. The potential energy surface of the nucleus ${ }^{32} \mathrm{Mg}$ in the $q$ direction is wider and flatter than the one for ${ }^{24} \mathrm{Mg}$ and steeper in the $\delta$ values.

Finally, we represent in Fig. 7 the same potential energy surfaces in the $q, \delta$ plane for the isotopes ${ }^{50} \mathrm{Ca},{ }^{52} \mathrm{Ca}$, and ${ }^{54} \mathrm{Ca}$, only for the PN-VAP and PN-VAP + PNAMP cases. Here we can study the evolution of the energy surfaces as a function of the neutron number. In the PN-VAP approach (left panels) all the three have a spherical ground state, owing to the $Z=20$ proton shell closure, the contours are steep in the $q$ coordinate, but rather soft in the $\delta$ coordinate for $\delta$ values less than about 3.5. Though one would expect a softening in the $q$ direction with increasing neutron number as an indication of the growth in collectivity we observe that this is not the case for ${ }^{52} \mathrm{Ca}$. As we see below this is attributable to a subshell closure. In the PN-VAP + PNAMP approach and for $I=0 \hbar$ (right panels), we observe the well-known effect of the AMP: the softening of the potential energy surface in the $q$ coordinate giving rise to the presence of a structure of two minima, one prolate and one oblate at -60 and $60 \mathrm{fm}^{2}$, respectively. The softness in the $\delta$ coordinate remains, however, unchanged.
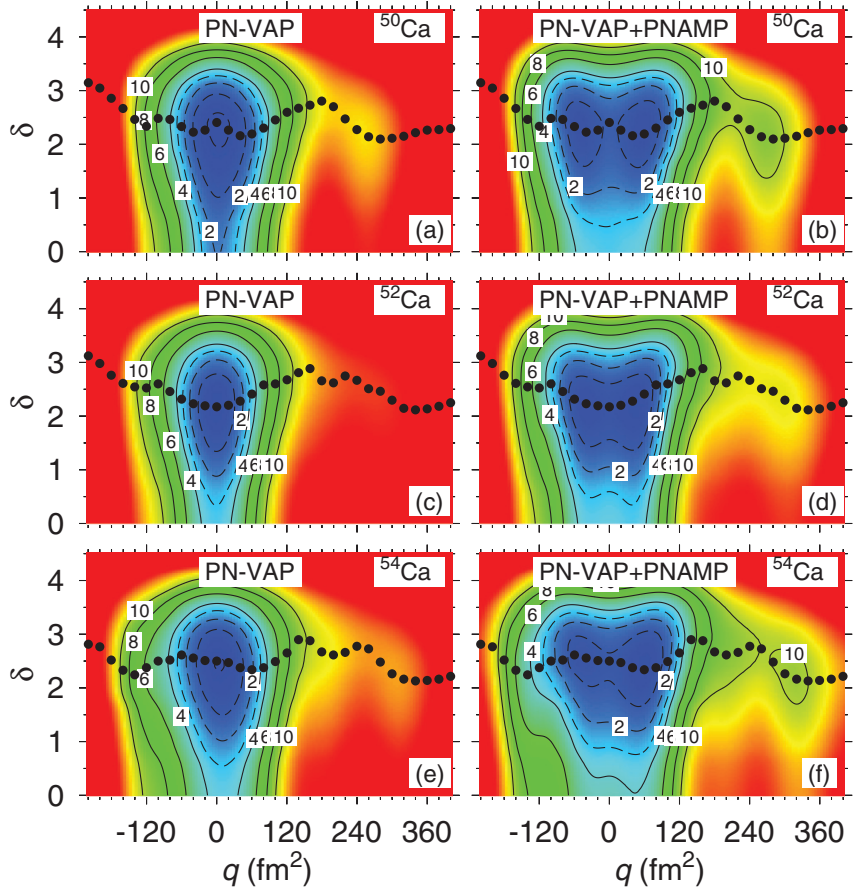

FIG. 7. (Color online) (Left panels) Potential energy surfaces for the nuclei ${ }^{50} \mathrm{Ca},{ }^{52} \mathrm{Ca}$, and ${ }^{54} \mathrm{Ca}$ in the PN-VAP approach. (Right panels) Potential energy surfaces for the same nuclei in the PN-VAP + PNAMP approach for $I=0$. In both cases the contours follow the same interval as in Fig. 5.

Another interesting issue is the fact that the 1D solutions of the PN-VAP approach are not only orthogonal to the contours of the corresponding 2D calculations, as they should, but, to a good approximation, they are also orthogonal to the contours of the PN-VAP + PNAMP approach. This indicates that, at least for $I=0 \hbar$, the PN-VAP 1D w.f.'s are, to a good approximation, a self-consistent solution of the 2D PN-VAP + PNAMP approach. One can check in Figs. 5 and 6 that for those nuclei, and in this approach, this is also the case. One can also realize in Fig. 5 that, to a lesser extent, this is also the case in the HFB case as compared with the HFB + AMP and that this is not the case, at least around the energy minima, in the HFB + PNAMP approach. These facts are, as we will see later, a clear advantage of the PN-VAP intrinsic w.f.

The conclusion of this section is that, in general and independently of the approach, nuclei are soft in the pairing degree of freedom in the small-to-moderately strong pairing regime; however, for the very strong pairing the potential energy becomes very steep.

\section{NORM MATRIX ELEMENTS}

Into the diagonalization of the HW equation [Eq. (5)] enter two quantities, the Hamiltonian overlap and the norm overlap. In the preceding section we discussed to some extent the diagonal matrix elements of the Hamiltonian. We now concentrate on the norm overlap to get further inside of the different approximations. The main differences in the norm overlaps of the different approaches appear between the PNP theories and the non-PNP ones. Furthermore, to disentangle 

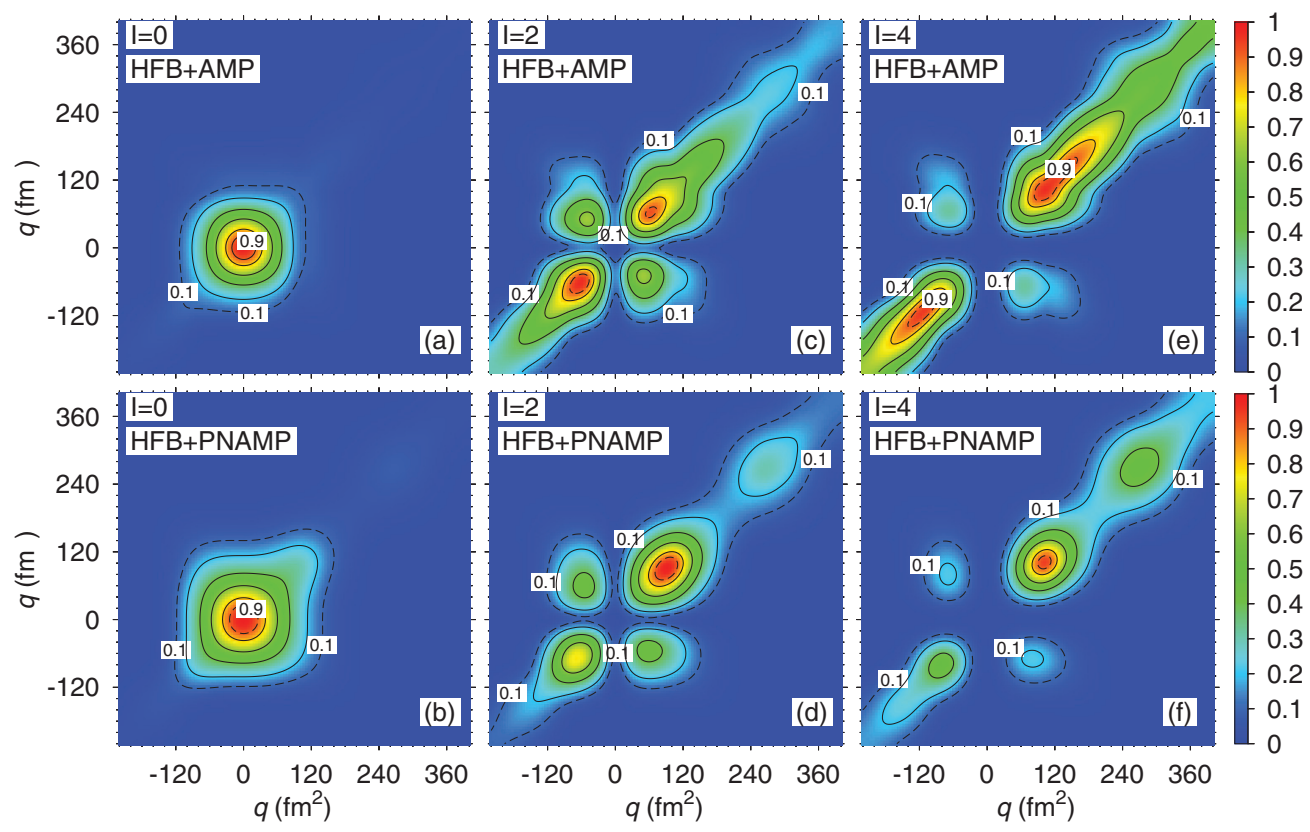

FIG. 8. (Color online) Renormalized norm overlaps of the ${ }^{52} \mathrm{Ti}$ nucleus. The original matrix elements have been divided by a factor to have in each panel the maximum value equal to unity. (Top panels) The HFB + AMP approach, for $I=0$ (factor $=1.0), I=2($ factor $=0.484$ ), and 4 (factor $=0.316$ ). (Bottom panels) The HFB + PNAMP, for $I=0$ (factor $=0.191), I=2$ (factor $=0.218)$, and 4 (factor $=0.202)$. The continuous lines represent contours from 0.2 to 0.8 in steps of 0.2 and in dashed lines we depict the 0.1 contour and the 0.9 one.

PNP effects from the ones stemming from VAP or PAV treatment we concentrate on the norms of the HFB + AMP approach and the HFB + PNAMP one. The norms of the PN-VAP + PNAMP are rather similar to the HFB + PNAMP ones and will not be shown here.

Because of the huge amount of nondiagonal elements if the 2D calculations, we analyze in detail the 1D case and some selected results in the $2 \mathrm{D}$ case.

In panel (a) of Fig. 8 we display contour plots of the norm overlaps $\left\langle\phi(q)\left|P^{I=0}\right| \phi\left(q^{\prime}\right)\right\rangle$ for the nucleus ${ }^{52} \mathrm{Ti}$ in the HFB + AMP approach for $I=0$ as a function of $\left(q, q^{\prime}\right)$. Because the HFB w.f.'s are normalized to unity, for the spherical case, i.e., for $q=q^{\prime}=0$, the norm overlap is just one. Taking this point as the center we observe a radial exponential decrease of the norm with $q$. For $q=90 \mathrm{fm}^{2}$ the norm is reduced to one-tenth of the value at $q=0 \mathrm{fm}^{2}$. In panel (b) of Fig. 8 we now display $\left\langle\phi(q)\left|P^{I=0} P^{N}\right| \phi\left(q^{\prime}\right)\right\rangle$, i.e., the norm matrix in the HFB + PNAMP. For $q=q^{\prime}=0$ this quantity is $\left\langle\phi(q)\left|P^{N}\right| \phi\left(q^{\prime}\right)\right\rangle$, which is not unity; as a matter of fact, it is 0.191 , because the norm decreases as more projectors are added. To compare the different norms we have divided all panels with the corresponding factors to have the maximum value equal to unity in all panels. The norm distribution in the HFB + PNAMP looks like the one for the HFB + AMP though more extended and stretched along the diagonal. For the $I=2$ case, panels (c) and (d), we find that, as expected, the norms are zero around the spherical shape, they are large around $|q|=\left|q^{\prime}\right| \approx 60 \mathrm{fm}^{2}$ (for the HFB + PNAMP case at slightly larger values) and decrease smoothly along the diagonal and the perpendiculars to the diagonal $\left(q+q^{\prime}=\right.$ const.). In the HFB + AMP case the distribution is practically symmetric with respect to the line $q=-q^{\prime}$; i.e., the prolate and oblate parts do have similar values for the norm. In the HFB + PNAMP this is not the case, we find a clear predominance of the prolate side. This is attibutable to the fact that this norm is sensitive to the pairing content of the w.f.'s. For $I=4$ the situation goes along the same lines as the $I=2$ case but with stronger differences. Besides the oblate-prolate asymmetry present in the HFB + PNAMP approach we also observe that the HFB + AMP norms are more extended for $I=2$ and 4 than the HFB + PNAMP. This is a clear evidence of particle-number mixing and of spurious correlations in the $\mathrm{HFB}+\mathrm{AMP}$ case.

In Fig. 9 we extend the analysis to the 2D case; i.e., we now concentrate on the matrix elements $\left\langle\phi(q \delta)\left|P^{I}\right| \phi\left(q^{\prime} \delta^{\prime}\right)\right\rangle$ (HFB + AMP approach) and $\left\langle\phi(q \delta)\left|P^{I} P^{N}\right| \phi\left(q^{\prime} \delta^{\prime}\right)\right\rangle$ (HFB + PNAMP approach) for $I=0$ and 2 and again for the nucleus ${ }^{52} \mathrm{Ti}$. We choose two $q$ deformations, namely, -80 and $100 \mathrm{fm}^{2}$, which approximately correspond to the values of the energy minima, and three $\delta$ values which represent the weak $(\delta=0.5)$, the medium $(\delta=2.0)$, and the strong $(\delta=3.5)$ pairing regime. ${ }^{2}$ Furthermore, we take $q^{\prime}=q$ in all the panels, the fixed $(q, \delta)$ values are given in each panel, and the variable $\delta^{\prime}$ is indicated in the abscissa of each panel. We present calculations for $I=0$ (first and third rows) and $I=2$ (second and fourth rows). As a reference for $I=0 \hbar$ we also present the HFB overlaps $\left\langle\phi(q \delta) \mid \phi\left(q^{\prime} \delta^{\prime}\right)\right\rangle$.

While for fixed $q$ the HFB and the HFB + AMP approaches present a more or less Gaussian behavior around the diagonal

\footnotetext{
${ }^{2}$ The terms weak, medium, and strong pairing regime are loosely used in reference to the domains where the diagonal matrix elements of the HFB and HFB + AMP peak.
} 


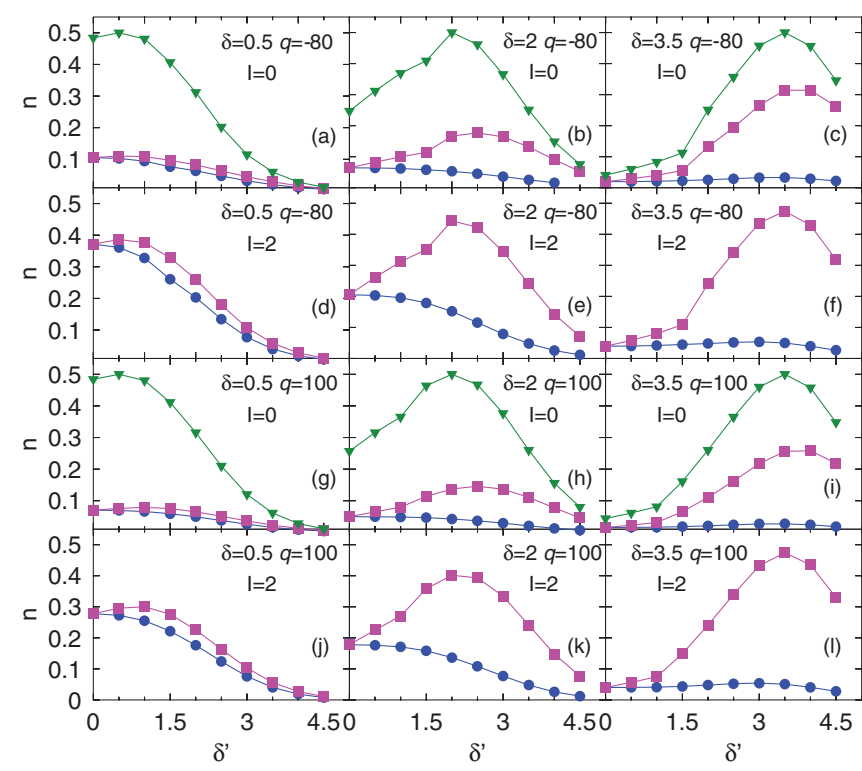

FIG. 9. (Color online) Norm matrix elements for ${ }^{52} \mathrm{Ti}$ for different $q$ and $\delta$ values (see main text) in the HFB + PNAMP (blue circles), $\mathrm{HFB}+\mathrm{AMP}$ (magenta squares), and HFB (green triangles and only for $I=0 \hbar$ ). Because the HFB w.f. are normalized to unity these norms are multiplied by 0.5 to make the pictures more legible.

matrix element $\delta^{\prime}=\delta$, the HFB + PNAMP does not. For a fixed $\delta$ value, the norm of this approach either decreases or remains more or less constant for increasing $\delta^{\prime}$. The reason is very simple: If we expand the intrinsic w.f. in eigenstates of the particle-number operator,

$$
\left.\left|\phi\left(q \delta^{\prime}\right\rangle=\sum_{\alpha^{\prime} N^{\prime}} C_{\alpha^{\prime} N^{\prime}}\left(q \delta^{\prime}\right)\right| \alpha^{\prime}, N^{\prime}\right\rangle,
$$

the HFB + PNP norm is given by

$$
\left\langle\phi(q \delta)\left|P^{N}\right| \phi\left(q \delta^{\prime}\right)\right\rangle=\sum_{\alpha} C_{\alpha N}(q \delta) C_{\alpha N}\left(q \delta^{\prime}\right) .
$$

Obviously, for $\delta^{\prime}=0, \sum_{\alpha}\left|C_{\alpha N}\left(q \delta^{\prime}\right)\right|^{2}=1$. For large $\delta^{\prime}$ the spread in the number of particles grows considerably and more terms contribute to the expansion (10). However, because

$$
\sum_{\alpha^{\prime} N^{\prime}}\left|C_{\alpha^{\prime} N^{\prime}}\left(q \delta^{\prime}\right)\right|^{2}=1
$$

for a given $N, \sum_{\alpha} C_{\alpha N}(q \delta) C_{\alpha N}\left(q \delta^{\prime}\right)$ will, in general, decrease with growing $\delta^{\prime}$. In this argument we have omitted the angularmomentum operator which could modulate the behavior of the norm.

If we now compare both approaches, the tendency is clear: In the small pairing regime (left panels), there are few differences between both approaches independent of the $q$ value; in the medium pairing regime (middle panels), we find larger deviations; and in the strong regime (right panels), the deviations are even larger. In the strong pairing regime the HFB + AMP curves for $I=0 \hbar$ look somewhat like the HFB one. Furthermore, we observe that the deviations of the HFB + AMP with respect to the HFB + PNAMP increase with the angular momentum. An interesting observation is the fact that whereas the HFB + PNAMP approach, in general, presents a mostly decreasing behavior with increasing $\delta^{\prime}$, the HFB + AMP one coincides with the HFB + PNAMP one at the small $\delta^{\prime}$ then increases with growing $\delta^{\prime}$ up to a maximum value for $\delta^{\prime}=\delta$, then decreases again and in the limit of very large $\delta^{\prime}$ would coincide with the HFB + PNAMP value. The last one is clearly the semiclassic limit. Unfortunately, large deviations take place between both approaches at the $\delta^{\prime}$ values which are the most common in nuclei.

\section{SPECTRA}

We discuss now the results of the SCCM calculations, for which the HW [Eq. (5)] has to be solved. Before discussing the excitation spectra we comment on the limitations of our approaches. In our description we are considering mainly collective degrees of freedom, namely, the quadrupole deformation and the pairing gap. Though we are considering different nuclear shapes and, in principle, single-particle degrees of freedom can be expanded as linear combinations of different configurations, we cannot claim to describe properly genuine single-particle states but only in an approximate way. Collective states, however, are very well described in our approach.

The HW equation has to be solved separately for each value of the angular momentum, the diagonalization of this matrix provides the yrast and the excited states, $I_{1}^{+}, I_{2}^{+}, I_{3}^{+}, \ldots$, for each angular momentum. These energy levels, normalized to the ground-state energy $\left(0_{1}^{+}\right)$, provide the spectrum of the nucleus. Again, we study the three cases on which we are focused, namely HFB + AMP, HFB + PNAMP, and PN-VAP + PNAMP. To evaluate the impact of the pairing fluctuations on the different observable we consider the solutions of the HW equation in 1D, with one coordinate $(q)$, and in $2 \mathrm{D}$, with two coordinates $(q, \delta)$. We have calculated the four lowest states for each angular momentum.

Though we are acquainted with the diagonal matrix elements of the HW equation through the energy surface contour plots previously discussed, the magnitude of the nondiagonal elements and thereby the energy gain after the solution of the HW equation depend on the approach. In general, because of self-consistency and owing to the quality of the approach before the HW diagonalization, we expect a smaller energy gain - as compared with the energy minimum of the potential energy - in the PN-VAP + PNAMP case than in the HFB + PNAMP one. For the special case of $I=0 \hbar$ we can make a thorough discussion of the different contributions because in this case we can solve the HW equation within our framework even for the plain HFB approach. The reason is that in a semiclassical approach to eigenstates of the angular momentum (cranking approach), one has to add the term $-\omega J_{x}$ to the variational principle [Eq. (2)]. The cranking frequency being determined by the condition $\left\langle J_{x}\right\rangle=\sqrt{I(I+1)}$. Because our HFB w.f.'s do not break time reversal, the condition $\left\langle J_{x}\right\rangle=0$ is always satisfied and the plain HFB approach can be considered to approximately describe $I=0 \hbar$ states.

In Fig. 10 and in Table II we display the absolute energy of the three first eigenvalues of the HW equation for $I=0 \hbar$ in different approaches and in the 1D and 2D cases. In the abscissa the different approaches are indicated; the line connecting them is used to guide the eye. The simplest approximation is 


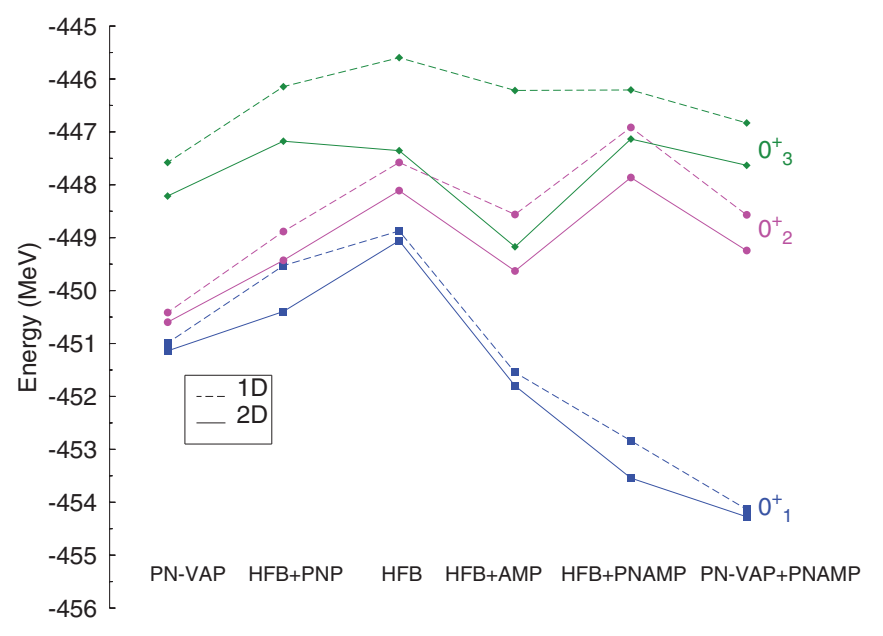

FIG. 10. (Color online) Evolution of the total energies of the ground-state energy $0_{1}^{+}$(blue squares) and excited energies $0_{2}^{+}$ (magenta circles) and $0_{3}^{+}$(green diamonds) as a function of the different approximations for ${ }^{52} \mathrm{Ti}$. Dashed lines correspond to $1 \mathrm{D}$ calculations and continuous lines are for 2D calculations.

provided by the solution of the HW equation with plain HFB w.f. without any kind of projectors. The other ones correspond to adding more complexity to the w.f.'s. The general behavior is as follows. (1) For a given approach the 2D approach is always lower than the 1D one. This is clear because within a given approach going from $1 \mathrm{D}$ to $2 \mathrm{D}$ means to add an extra degree of freedom. (2) The ground-state energy always decreases with increasing complexity of the w.f. If we now concentrate on the HFB states we observe that by a separate PNP (left of the HFB solution) or an AMP (right) one obtains, in general, an energy decrease; however, when a simultaneous PNAMP is performed an interference effect appears (see states $\mathrm{O}_{2}^{+}$and $\mathrm{O}_{3}^{+}$in $1 \mathrm{D}$ and 2D) and as a consequence some states increase its energy as compared with the simpler approach. We also observe that, as expected, in the VAP approaches (PN-VAP and PN-VAP + PNAMP) the energy gain from 1D to $2 \mathrm{D}$ is smaller than in the HFB approaches (HFB + PNP and $\mathrm{HBF}+\mathrm{PNAMP}$ ). We can also appreciate in the two most left (right) approaches the difference between a VAP and a PAV

TABLE II. Absolute energies of the three lowest states in several approaches for ${ }^{52} \mathrm{Ti}$, in $\mathrm{MeV}$.

\begin{tabular}{lcccc}
\hline \hline & & $0_{1}^{+}$ & $0_{2}^{+}$ & $0_{3}^{+}$ \\
\hline PN-VAP & 1D & -450.991 & -450.415 & -447.579 \\
& 2D & -451.140 & -450.598 & -448.213 \\
HFB + PNP & 1D & -449.528 & -448.883 & -446.145 \\
& 2D & -450.395 & -449.428 & -447.176 \\
HFB & 1D & -448.872 & -447.578 & -445.598 \\
& 2D & -449.056 & -448.111 & -447.355 \\
HFB + AMP & 1D & -451.547 & -448.561 & -446.217 \\
HFB + PNAMP & 2D & -451.800 & -449.629 & -449.173 \\
& 1D & -452.837 & -446.915 & -446.208 \\
PN-VAP + PNAMP & 2D & -453.543 & -447.863 & -447.135 \\
& 2D & -454.136 & -448.570 & -446.832 \\
\hline \hline
\end{tabular}

approach for the particle-number case and for a given 1D or $2 \mathrm{D}$ case. For the ground state and in the 1D case the energy gain of a VAP as compared to a PAV approach amounts to 1.5 MeV, the same quantity but now with AMP amounts to 1.3 MeV. Finally, we mention that the total energy gain of the $0_{1}^{+}$state from the simplest $\mathrm{HFB}(1 \mathrm{D})$ description to the most sophisticated one PN-VAP + PNAMP(2D) amounts to $5.4 \mathrm{MeV}$.

We now look for the excitation spectra, but before making a detailed description let us just mention a very general argument to guide our discussion. The comment above on the cranking approximation can also be interpreted in the light of a quantum approximation to an angular-momentum VAP method. According to the Kamlah expansion [40], a VAP of the angular momentum can be approximated, to first order, in the following way: The intrinsic HFB w.f., $|\phi\rangle$, is determined by minimizing the energy $E^{\prime}=\langle\phi|\hat{H}| \phi\rangle-\omega\left\langle\phi\left|\hat{J}_{x}\right| \phi\right\rangle$ with $\omega$ determined by the constraint $\left\langle J_{x}\right\rangle=\sqrt{I(I+1)}$; the energy is provided by $E^{I}=\left\langle\phi\left|\hat{H} P^{I}\right| \phi\right\rangle /\left\langle\phi\left|P^{I}\right| \phi\right\rangle$. Because for $I=$ $0 \hbar,\left\langle\phi\left|\hat{J}_{x}\right| \phi\right\rangle=0$, the Kamlah prescription applies in this case in the three approaches, but for $I \neq 0 \hbar$ this is not the case because our w.f.'s do not break time reversal and they cannot fulfill the constraint on the angular momentum. That means that our approaches favor the states with $I=0 \hbar$ because for them an approximate VAP for the angular momentum is performed. For $I \neq 0 \hbar$ this is not the case and we just do plain PAV. From these arguments and from this perspective it is obvious that the quality of the approach diminishes with growing $I$ values. That means the relative energy gain will be largest for $I=0 \hbar$, and for $I \neq 0 \hbar$ it will comparatively decrease with increasing $I$. Thus, in our current approach we predict stretched spectra; this will not be the case anymore if we break the time-reversal symmetry [41].

In Fig. 11 we present the excitation spectrum for ${ }^{52} \mathrm{Ti}$ in our three basic approaches and in the 1D and 2D calculations. The levels are ordered just by the energy. In the left-hand part we display the most complete approach, namely the PN-VAP + PNAMP. The general trend is that the $1 \mathrm{D}$ calculation is more stretched than the $2 \mathrm{D}$ one. This is a clear manifestation of the following fact: Because the $1 \mathrm{D}$ and the $2 \mathrm{D}$ calculations are self-consistent the ground-state energy before the HW diagonalization, i.e., the minimum of the potential energy surfaces, is the same in both calculations and even after the HW diagonalization they are rather similar; see Table II. This result is a consequence of the fact that the variational principle used to determine the w.f.'s $|\phi\rangle$ favors ground states. In the $1 \mathrm{D}$ calculations there is no room for the excited states to change the pairing content of a given w.f.; however, in the 2D calculations the flatness of the pairing degree of freedom opens the possibility of choosing different pairing energies for a given deformation $q$, allowing thereby an energy lowering. We see, therefore, that the consideration of additional degrees of freedom partially compensates the above-mentioned problem of approximate VAP for $I=0 \hbar$ versus PAV for $I \neq 0 \hbar$. In reality, we are doing a restricted VAP; see Ref. [42] for more details.

In the middle of Fig. 11 the HFB + PNAMP spectrum is presented. This spectrum is, in general, more stretched than the PN-VAP + PNAMP one. Another difference is the fact 


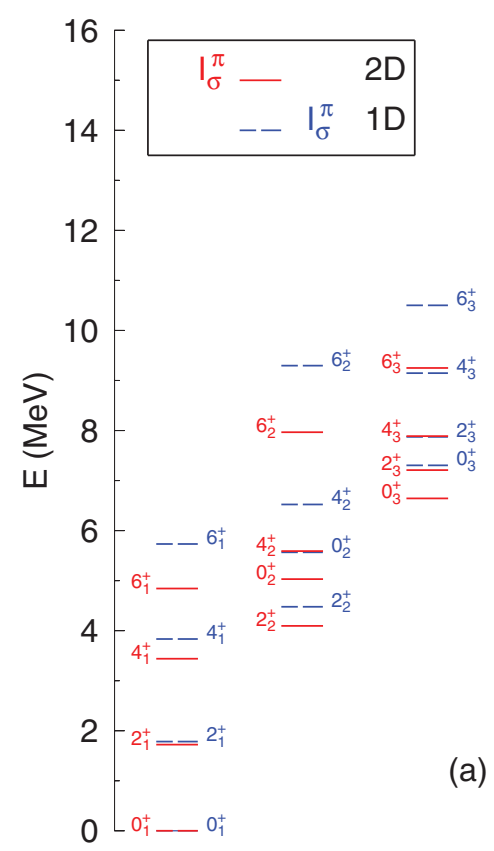

PN-VAP+PNAMP

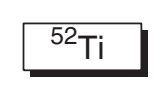

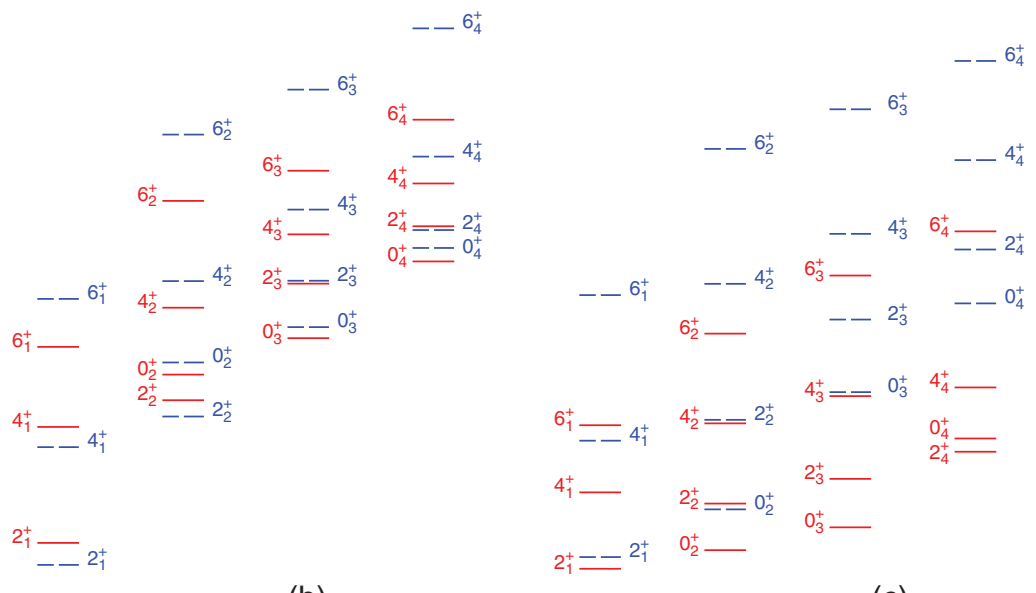

(b)

(c)
$0_{1}^{+}-0_{1}^{+}$

$\mathrm{HFB}+\mathrm{AMP}$

FIG. 11. (Color online) Spectra of ${ }^{52} \mathrm{Ti}$ in the PN-VAP + PNAMP (left), HFB + PNAMP (middle), and HFB + AMP (right) approaches. The four lowest states for spin $0^{+}, 2^{+}, 4^{+}$, and $6^{+}$are represented in $1 \mathrm{D}$ (dashed lines) and 2D (continuous lines).

that the ordering of some levels, in particular the yrast ones, of the $1 \mathrm{D}$ and $2 \mathrm{D}$ calculations are inverted as compared with the PN-VAP + PNAMP one. The reason for this behavior is the lack of self-consistency (in the sense of the end of Sec. IV B) of this approach. As we can see in the panel (e) of Fig. 5, the path of the 1D solution in the $(\delta, q)$ plane, i.e., the line of circles, goes along lines of smaller pairing correlations than the minima displayed by the $2 \mathrm{D}$ contour plots. Consequently, in 1D the mass parameter associated with the collective motion is larger than in $2 \mathrm{D}$ and the associated spectrum more compressed in the former than in the latter one. This effect combined with the additional degree of freedom of the 2D discussed above makes that only the lower levels are inverted.

Finally, in the right part of Fig. 11 the HFB + AMP approach is displayed. First, we observe very much compressed spectra as compared with the other approaches. The fact that all states with the same spin are much closer to each other than in the PNP approaches is remarkable. One furthermore notices the unusually large lowering of the $2 \mathrm{D}$ states as compared with the 1D ones. These facts seems to indicate that, as mentioned in Sec. V, there is too much mixing in the solution of the HW equation owing to spurious contributions stemming from the nonconservation of the particle-number symmetry. One also observes that, contrary to the inversion of the HFB + PNAMP, the inversion of the 1D and 2D levels does not take place in this case. This is attributable to the fact that in this case we are more self-consistent than in the HFB + PNAMP case.

Concerning the 2D spectra in the three approaches one can understand the degree of compression of the spectra by looking at the righthand panels of Fig. 5. We observe that, by far, the softest surface towards small pairing correlations is the HFB + AMP, then, though to a lesser extent, PN-VAP + PNAMP (in the energetic relevant part, i.e., around the minima), and finally HFB + PNAMP relatively close to the former one. Correspondingly, we expect the HFB + AMP spectrum to be the most compressed, followed by PN-VAP + PNAMP and finally HFB + PNAMP relatively close to the latter one.

In Fig. 12 we display another example of HFB + AMP spectrum versus the PN-VAP + PNAMP one, this time for the deformed collective nucleus ${ }^{32} \mathrm{Mg}$. Though clear differences are observed between both spectra, especially for the $I_{3}$ and $I_{4}$ states, in the $I_{2}$ states the difference, at least for the lowest ones, is not as large as for the titanium case. In the PN-VAP + PNAMP case the whole spectrum is compressed because ${ }^{32} \mathrm{Mg}$ is very collective, as on can see in the broad potential displayed in Fig. 6, and rather steep in the pairing fluctuations. For the nucleus ${ }^{24} \mathrm{Mg}$ (not shown here), however, we find large differences between both approaches. Of course, in the latter one and in the HFB approach the pairing correlations vanish at the potential minimum (see Fig. 6), inducing a large moment of inertia and the corresponding compression of the HFB + AMP spectrum. In this case is difficult to disentangle both effects, i.e., the differences caused by the smaller pairing correlations (as compared with the PN-VAP) and the spurious mixing caused by the nonconservation of the particle number.

Last, we present in Fig. 13 the spectra of the isotopes ${ }^{50-52-54} \mathrm{Ca}$ in the PN-VAP + PNAMP to see the evolution of the spectra with the neutron number. We observe shifts in the $2 \mathrm{D}$ calculations with respect to the $1 \mathrm{D}$ with the same tendencies as the nuclei discussed above. In ${ }^{50} \mathrm{Ca}$ we observe 


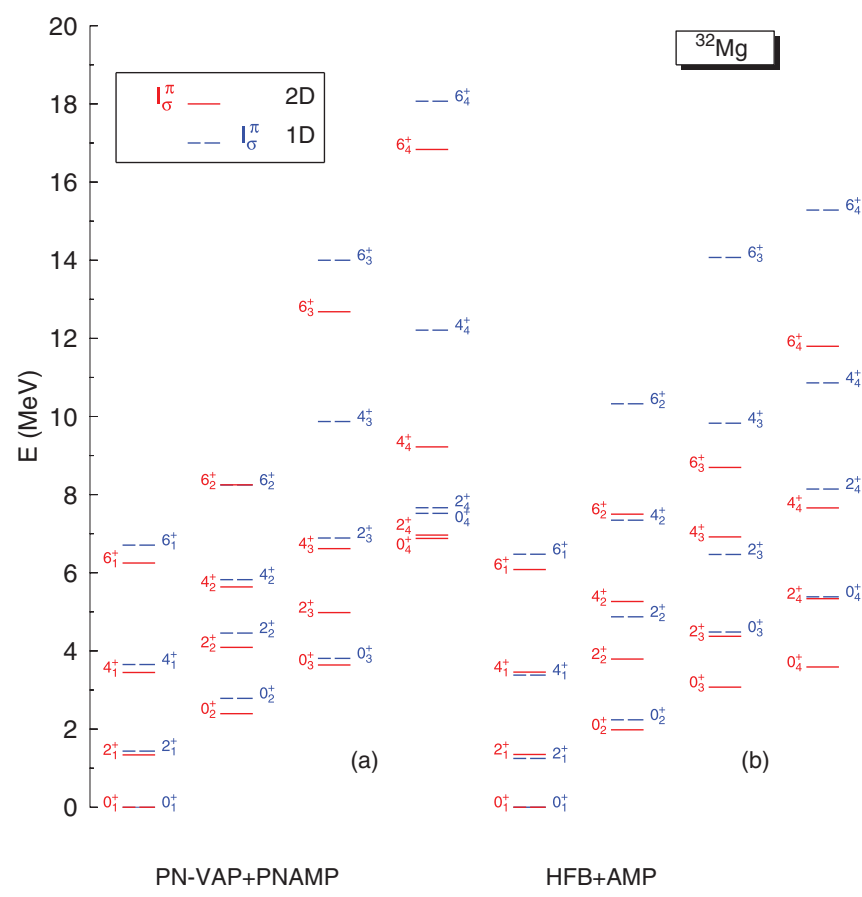

FIG. 12. (Color online) Spectra of ${ }^{32} \mathrm{Mg}$ in the PN-VAP + PNAMP approach (left) and the HFB + AMP approach (right).

an inversion of the $0_{3}^{+}$and $0_{4}^{+}$levels with the respective $2_{3}^{+}$and $2_{4}^{+}$levels that does not show up in ${ }^{52-54} \mathrm{Ca}$. In principle, one would expect an increase in collectivity with growing neutron number. Looking at the spectra we find that this not the case, the nucleus ${ }^{52} \mathrm{Ca}$ does not appears as a smooth interpolation of ${ }^{50} \mathrm{Ca}$ and ${ }^{54} \mathrm{Ca}$; as a matter of fact, the low-lying states of ${ }^{52} \mathrm{Ca}$ are higher in energy than in ${ }^{50-54} \mathrm{Ca}$. We observe, in particular, that the $2_{1}^{+}$state of the nucleus ${ }^{52} \mathrm{Ca}$ is higher in energy than in its neighbors; this fact has been interpreted as a subshell closure at $N=32$. The discussions going on [43-45] about the hypothetical shell closures at $N=32$ and $N=34$ have been settled by a recent measurement of the excitation energy of the $2_{1}^{+}$level in ${ }^{54} \mathrm{Ca}$ [46]. Our prediction for this state taking the pairing degree of freedom into account is in agreement with the experimental finding; see also Ref. [45].

We conclude this section by stressing the impact of the pairing of the nuclear spectra and the relevance of the PNP to avoid unwanted mixing.

\section{COLLECTIVE WAVE FUNCTIONS}

In this section we discuss the collective w.f.'s [see Eq. (6)] solution of the HW equations in $1 \mathrm{D}, g(q)$, and $2 \mathrm{D}, g(q, \delta)$, and in the three basic approaches only for the nucleus ${ }^{52} \mathrm{Ti}$.

In Fig. 14 we present the HW w.f.'s of the ${ }^{52} \mathrm{Ti}$ nucleus in the $1 \mathrm{D}$ case in the three basic approaches. The corresponding potential energy curves have been plotted in Fig. 4. In the left top panel of Fig. 4 the PN-VAP + PNAMP potential energy curve displays two quasi-coexistent minima, the lowest one prolate and the other one oblate; consequently, the w.f.'s [see Fig. 14(a)] of the $0_{1}^{+}$and $0_{2}^{+}$states display a two-hump structure with maxima (or maximum and minimum) at these values, the $0_{2}^{+}$with a node as one would expect for a $\beta$ vibration. The $0_{3}^{+}$state, however, peaks at large deformations in the prolate and the oblate potential shoulders and it has a two-node structure. In the HFB + PNAMP approach, the potential energy curve (see Fig. 4) presents also two minima,

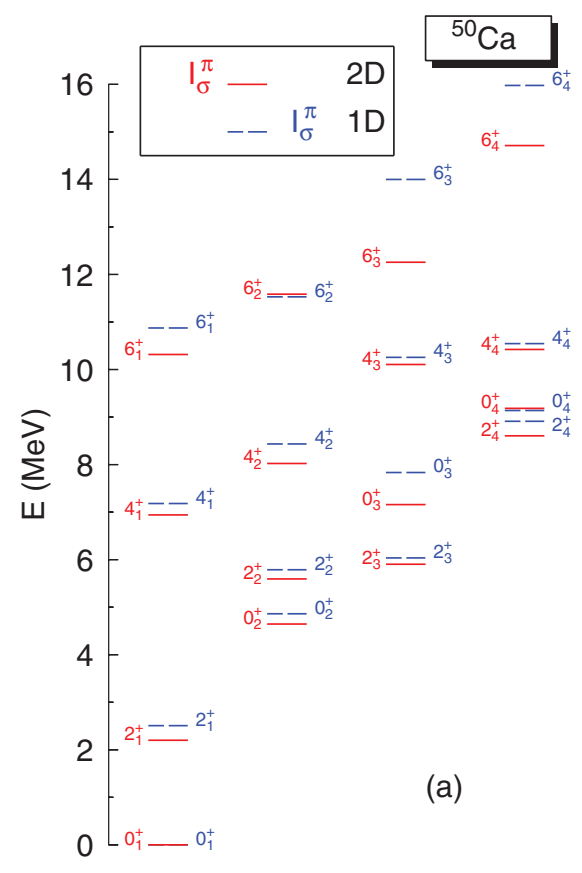

PN-VAP+PNAMP

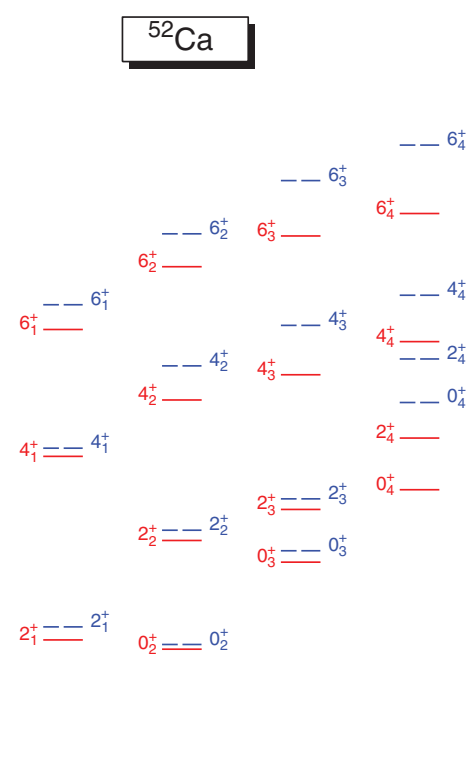

(b)

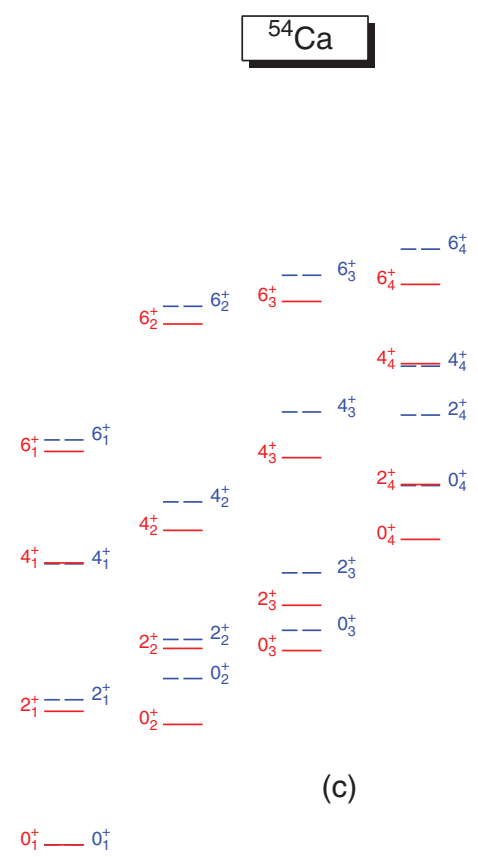

PN-VAP+PNAMP

FIG. 13. (Color online) Spectra of ${ }^{50} \mathrm{Ca},{ }^{52} \mathrm{Ca}$, and ${ }^{54} \mathrm{Ca}$ in the PN-VAP + PNAMP approach. 


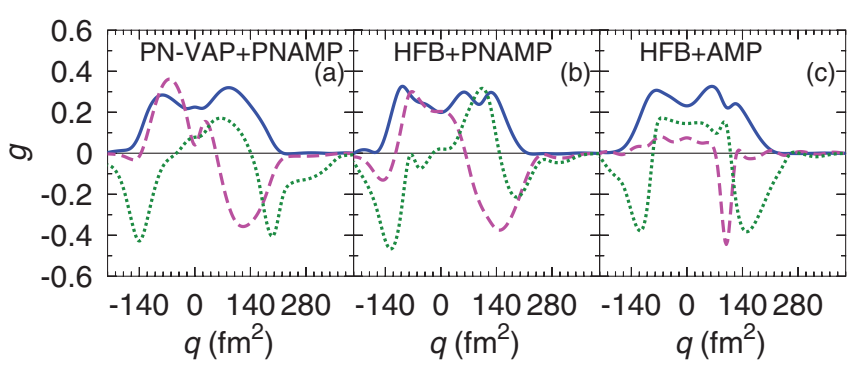

FIG. 14. (Color online) The w.f.'s of the lowest $0^{+}$collective states for the nucleus ${ }^{52} \mathrm{Ti}$ in various approaches: (left panel) in the $\mathrm{PN}-\mathrm{VAP}+\mathrm{PNAMP}$ approach, (middle panel) in the HFB + PNAMP approach, and (right panel) in the HFB + AMP approach in the 1D calculations. $0_{1}^{+}$continuous blue line, $0_{2}^{+}$dashed magenta line, and $\mathrm{O}_{3}^{+}$dotted green line. The w.f. of the $0_{2}^{+}$state in the HFB + AMP approach has been multiplied by a factor 0.5 .

although not as pronounced as in the previous case and somewhat narrower. The kink around $100 \mathrm{fm}^{2}$ has its origin in the neutron pairing energy collapse of the HFB w.f. at this point (see the right top panel of Fig. 4), and it causes the split of the prolate bump of the $0_{1}^{+}$state. The w.f. of the $0_{2}^{+}$state, similarly to the PN-VAP + PNAMP case presents a two-bump structure. The $0_{3}^{+}$one, though similar to the PN-VAP + PNAMP too, shows a decrease of the prolate shoulder, in part owing to the collapse of the pairing energy of the HFB w.f. at this point, as well as the fact that the magnitude of the shoulder in the potential energy is smaller than in the former case. As is well known at the energy minima the level density decreases and so does the pairing energy in the HFB approach (in some cases it even collapses); in a PN-VAP approach, obviously, this is not the case. In the HFB + AMP case the potential energy has the same two minima structure and it is even narrower than before. The w.f. of the $0_{1}^{+}$state has the right-hand side of the split bump smaller as compared with the former case. The big difference appears in the $0_{2}^{+}$state where the w.f. rises at the place where the neutron pairing gap gets zero. The $0_{3}^{+}$state, however, peaks at smaller prolate $q$ values owing to the near disappearance of the shoulder and the fact that the potential energy is somewhat narrower.

Concerning the $(q, \delta)$ calculations, the potential energy surfaces have been already discussed in Fig. 5, the 2D w.f.'s are presented in Fig. 15. We start again with the PN-VAP + PNAMP case. In panel (a) the contours lines of the w.f. of the $0_{1}^{+}$state are shown. In strong correspondence with the bottom right panel of Fig. 5 it presents a two-bump structure, rather soft in the pairing degree of freedom, with a predomination of the prolate side. The bump maxima are located at $q$ values close to the 1D case and centered at $\delta$ values close to the self-consistent solution (see circles in Fig. 5). The $0_{2}^{+}$state, panel (b), displays also a two-bump structure, this time with the maximum in the oblate side and soft in $\delta$. The maxima are located at $\delta$ values smaller than the $0_{1}^{+}$ state. It presents a nodal line at $q \approx 50 \mathrm{fm}^{2}$ as correspond to a $\beta$ vibration in $2 \mathrm{D}$. The $0_{3}^{+}$state, panel (c), presents a three-peak structure, two at large deformations and large pairing correlations and a smaller one around $80 \mathrm{fm}^{2}$ with smaller pairing correlations. This situation is similar to the 1D case where at similar $q$ values the same peaks are found. The fact that the large deformation peaks do have strong pairing correlations is attributable to the fact that the level density is very high at these deformations and that the $2 \mathrm{D}$ calculations allow that a given $q$ value can take different pairing content for different collective states.

Looking at panel (e) of Fig. 5, the 1D plots of Fig. 14 and taking into account the discussion above one can interpret the $2 \mathrm{D}$ w.f.'s of the HFB + PNAMP approach very easily. The main difference with the former case is that the $\beta$ vibration and the $0_{3}^{+}$state in this case are not as pure as in the PN-VAP + PNAMP case.

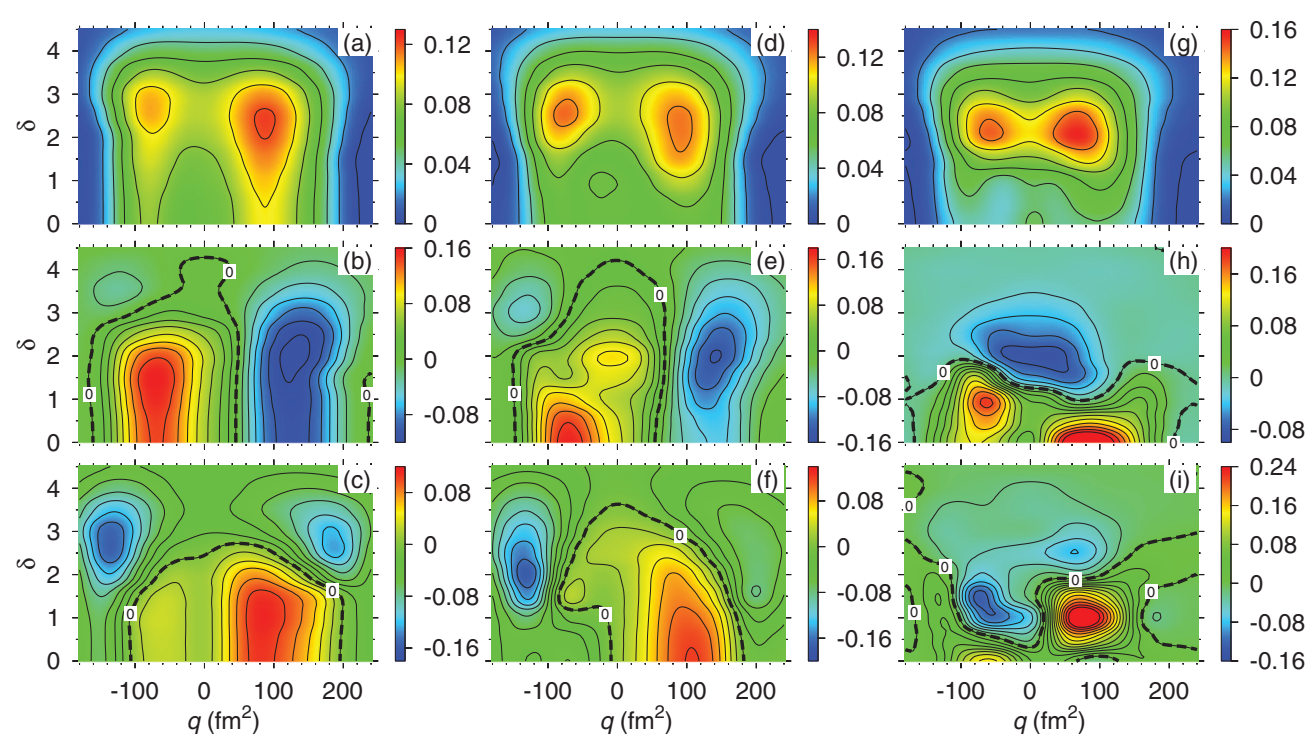

FIG. 15. (Color online) Contour lines of the w.f.'s of the three lowest $0^{+}$states of ${ }^{52} \mathrm{Ti}$ in different approaches in the $2 \mathrm{D}$ calculations. The step size is 0.02 for the states. The thick dashed lines correspond to the zeros of the w.f. To get larger resolution the $x$ axis runs from $-180 \mathrm{fm}^{2}$ up to $240 \mathrm{fm}^{2}$ at variance with former figures. 
The HFB + AMP collective w.f.'s look more different than the ones of the two former approximations. The $0_{1}^{+}$one still maintains the two-bump structure though more pronounced than in the HFB + PNAMP case. The two peaks are located at smaller $\delta$ values and closer to each other in the $q$ coordinate. The strong peak of $0_{2}^{+}$at $q \approx 110 \mathrm{fm}^{2}$ of the $1 \mathrm{D}$ case is also present in the $2 \mathrm{D}$ one but now a second peak appears at $q \approx$ $-60 \mathrm{fm}^{2}$. The $0_{3}^{+}$w.f. resembles the $1 \mathrm{D}$ case in some aspects. One has the impression that the $0_{2}^{+}$and $0_{3}^{+}$states of the $1 \mathrm{D}$ and of the other 2D PNP approaches get mixed in the HFB + AMP approach. In this approach the w.f.'s are more concentrated than in the two former ones. One can quantify this effect, noticing that the maxima (in absolute value) of the w.f. in the PN-VAP + PNAMP are $0.125,0.158$, and 0.198 for the $0_{1}^{+}$, $0_{2}^{+}$, and $0_{3}^{+}$, respectively. For the HFB + PNAMP the maxima are $0.133,0.183$, and 0.295 , in the same order, and for the HFB + AMP, they are 0.161, 0.200, and 0.357. The trend is clear: The concentration of the w.f. increases as the quality of the respective approach decreases.

Interestingly, though the potential energy surfaces in the three cases are rather similar [see panels (b), (e), and (f) of Fig. 5], the w.f.'s of the HFB + AMP are rather different from the other ones. This has obviously to do with the nondiagonal elements of the Hamilton overlap and the norm overlap. The former is through the dynamical corrections and the latter is through the linear dependence of the basis states.

We conclude this section again underlining the relevance of the PNP for a proper description of the properties of atomic nuclei.

\section{PAIRING VIBRATIONS}

One could also raise the question about the existence of pure pairing vibrations. They are on their own very interesting and a simultaneous study of the shape and pairing fluctuations will allow us to disentangle if there exist genuine pairing vibrations or they are washed out because of the energy predominance of the quadrupole ones. In this section we discuss this issue in the framework of the PN-VAP + PNAMP approach.

Pairing vibrations appear naturally when $1 \mathrm{D}$ calculations are performed using $\delta$ as the generating coordinate and fixing the deformation. In the top panels of Fig. 16, we show the potential energy (in black) and the collective w.f.'s [Eq. (6)] for the states $0_{1}^{+}$(blue) and $0_{2}^{+}$(magenta) as a function of $\delta$ at a fixed value of $q=-80 \mathrm{fm}^{2}$ and $q=80 \mathrm{fm}^{2}$ for the nucleus ${ }^{52} \mathrm{Ti}$. The ground-state w.f. does not present any node while the first excited state, $0_{2}^{+}$, shows one node as corresponds to a pairing vibration.

We now consider simultaneously the pairing and the quadrupole deformation degrees of freedom. The corresponding potential energy contour plot has been already discussed in Fig. 5. Because we expect vibrations associated to the deformation $(q)$ and with the pairing $(\delta)$ we now have to consider at least three states, the ground and the two lowest excited states, to seek for the shape and the pairing vibrations. In the left panels of Fig. 15 we have shown the w.f.'s [Eq. (6)] of the lowest three states. Because we have two coordinates we expect that the nodes of the w.f.'s of the 1D case will now turn to nodal lines. The ground-state w.f.'s [see panel
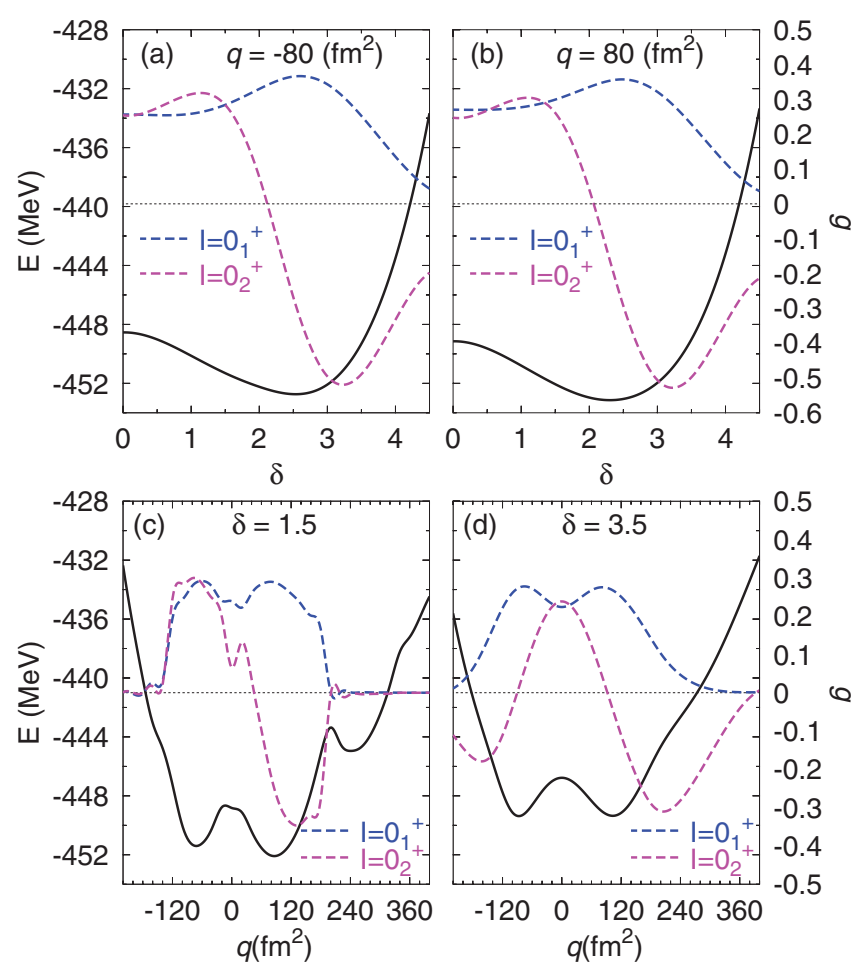

FIG. 16. (Color online) Potential well and w.f.'s of the two lowest states in $1 \mathrm{D}$ calculations for the nucleus ${ }^{52} \mathrm{Ti}$. (Top) For fixed $q$ value and with $\delta$ as a generator coordinate, (left panel) $q=-80 \mathrm{fm}^{2}$ and (right panel) $q=+80 \mathrm{fm}^{2}$. (Bottom) For fixed $\delta$ value and with $q$ as a generator coordinate, (left panel) $\delta=1.5$ and (right panel) $\delta=3.5$.

(a)] is everywhere positive; it has two maxima in a direct correspondence with the two minima of the potential well. The first excited state presents a maximum at $q \approx-60 \mathrm{fm}^{2}$ and a minimum at $q \approx+140 \mathrm{fm}^{2}$ with a nodal line in between at $q \approx 50 \mathrm{fm}^{2}$. This state is clearly identified as a $\beta$ vibration. In panel (c) the second excited state is depicted. It has two strong minima at large $q$ values and a maximum at $q \approx 80 \mathrm{fm}^{2}$ with a long tail towards the oblate side. The node line in this case is formed by two segments perpendicular to the $q$ axis and a curved segment more or less perpendicular to the $\delta$ axis. The two first segments would be an indication of a two-phonon $\beta$ vibration and the latter one of a pairing vibration.

To disentangle the role of the deformations and pairing vibrations we have performed two $1 \mathrm{D}$ calculations to decouple these degrees of freedom. In the first one we do not consider the interaction of different nuclear shapes; i.e., we assume

$$
\left\langle q_{1} \delta_{1}\left|H \hat{P}^{N} \hat{P}^{I}\right| q_{2} \delta_{2}\right\rangle=\left\langle q_{1} \delta_{1}\left|H \hat{P}^{N} \hat{P}^{I}\right| q_{1} \delta_{2}\right\rangle \delta_{q_{2} q_{1}} .
$$

For a Schrödinger-type equation this would amount to diagonalize separately each $q$ value. For a HW-type equation, however, additionally one has to assume

$$
\left\langle q_{1} \delta_{1}\left|\hat{P}^{N} \hat{P}^{I}\right| q_{2} \delta_{2}\right\rangle=\left\langle q_{1} \delta_{1}\left|\hat{P}^{N} \hat{P}^{I}\right| q_{1} \delta_{2}\right\rangle \delta_{q_{2} q_{1}}
$$

to have that equivalence. In any case, what we do is to perform separately, for each of the $32 q$ values of our 2D GCM basis, a 1D GCM calculation with $\delta$ as generator. The two top plots of Fig. 16 correspond to two of such calculations. Now we take the lowest state of each of the the 32 values, i.e., the $0_{1}^{+}$ 


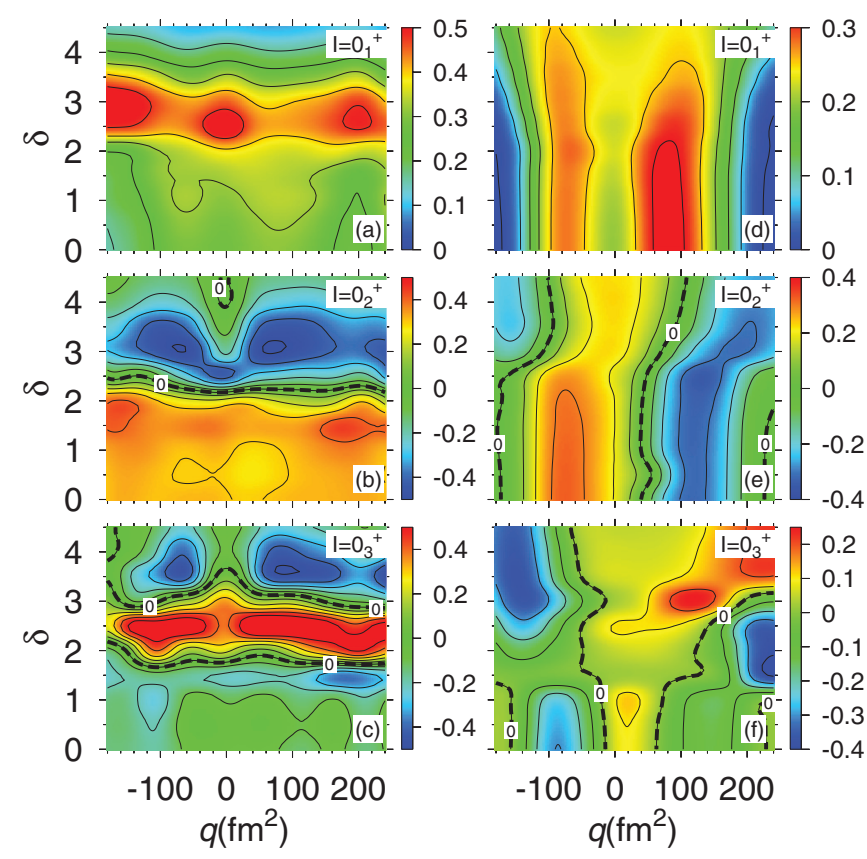

FIG. 17. (Color online) Wave functions of the lowest $0^{+}$states of ${ }^{52} \mathrm{Ti}$ of combined 1D generator coordinate calculations; see main text for further explanations. (Left) Calculations for a fixed $q$ value taking $\delta$ as generator coordinate. (Right) Calculations for a fixed $\delta$ value taking $q$ as generator coordinate.

state, and merge their w.f.'s in a 2D plot. The result of drawing contour plots is displayed in panel (a) of Fig. 17. The same is done with the $0_{2}^{+}$and $0_{3}^{+}$w.f.'s and plotted in the panels (b) and (c) of Fig. 17, respectively. The horizontal node line in the $0_{2}^{+}$plot [panel (b)] indicates that we have to do with a genuine pairing vibration. The plot in panel (a) with no node line corresponds to the ground state and the one in panel (c) to a two-phonon pairing vibration.

We now perform the other 1D calculation. In this case we do not allow states with different pairing energies to interact; that means

$$
\left\langle q_{1} \delta_{1}\left|H \hat{P}^{N} \hat{P}^{I}\right| q_{2} \delta_{2}\right\rangle=\left\langle q_{1} \delta_{1}\left|H \hat{P}^{N} \hat{P}^{I}\right| q_{2} \delta_{1}\right\rangle \delta_{\delta_{2} \delta_{1}},
$$

and we have to do with 1D calculations, for the ten fixed $\delta$ values of our basis, and the quadrupole degree as generator coordinate. In the bottom panels of Fig. 16 we show two such calculations, namely for $\delta=1.5$ and $\delta=3.5$. We observe that neither the potentials nor the w.f.'s are harmonic. As before, we merge the 10 w.f.'s in a $2 \mathrm{D}$ plot for the $0_{1}^{+}, 0_{2}^{+}$, and $0_{3}^{+}$ states, as depicted in the panels (d), (e), and (f) of Fig. 17, respectively. Again the node lines are indicative of zero, one, and two vibrational $\beta$ phonons.

The goal is to learn from the comparison of the fake 2D w.f. of Fig. 17 and the real ones of Fig. 15, but a consideration must be done first. In the plots of the fake 2D w.f.'s the normalization of the w.f.'s is different than in the real 2D calculations. For fixed $q, \sum_{\delta}|g(q \delta)|^{2}=1$, and for fixed $\delta, \sum_{q}|g(q \delta)|^{2}=1$, while in the full $2 \mathrm{D}$ calculations $\sum_{\delta, q}|g(q \delta)|^{2}=1$. That means the w.f.'s will be transversally (longitudinally) extended in the calculation at fixed $\delta(q)$. This explains, for instance, the presence of strength at very large pairing correlations for the $\delta$ fixed calculations. A look at the lower panels of Fig. 16 shows that the potential energy of the $\delta=3.5$ value is a few $\mathrm{MeV}$ higher in energy than the $\delta=1.5$, indicating that as soon as pairing correlations are switched on the w.f. will be inhibited for very large $\delta$ values. That means in these plots the energy consideration which will favor (hinder) some values of $q$ or $\delta$ is not taken into account. For instance, maxima of the w.f.'s with very large values of $\delta$ are highly unlikely to prevail.

We now proceed to compare these plots with the w.f.'s of the full 2D calculations displayed in the left panels of Fig. 15. The ground state of the full 2D calculations [see panel (a)] presents two maxima, a big one at $+80 \mathrm{fm}^{2}$ and a smaller one at $-80 \mathrm{fm}^{2}$. The bulk structure of this constellation is found in the ground state of the calculations with $q$ as a generator coordinate; see panel (d) of Fig. 17. We notice here that, as mentioned, the maxima are more extended in the $\delta$ coordinate than the former ones. The ground-state w.f. with the pairing correlations as generator coordinate [see panel (a) of Fig. 17] is, however, completely different. It displays two maxima centered at very large deformations, $\pm 200 \mathrm{fm}^{2}$ corresponding to the large level density which favors large pairing correlations and one at $q=0$. This w.f. has little in common with the real 2D w.f. However, looking at this w.f. at small and large pairing correlations, we can easily imagine that the consideration of the pairing interaction will modulate the ground-state w.f. of the fixed $\delta$ calculations of panel (d).

The $0_{2}^{+}$state of the full 2D calculations [see panel (b) of Fig. 15] from the node structure point of view unequivocally resembles the $0_{2}^{+}$of the calculations with $q$ as generator coordinate [see panel (e) of Fig. 17], except for the artificial extension for large $\delta$ mentioned above.

The genuine pairing vibration of panel (b) of Fig. 17 does not have, however, a direct counterpart in the second excited state of the full 2D calculations shown in panel (c) of Fig. 15. However, if we look at the self-consistent 1D calculations of Fig. 14(a), we observe that the peak structure of the $0_{3}^{+}$state (dotted line) is very similar to a cut along $\delta=2.5$ in panel (f) of Fig. 17. The value $\delta=2.5$ is not arbitrary because it corresponds approximately to the self-consistent path in panel (f) of Fig. 5. The state $0_{3}^{+}$of Fig. 17(f) corresponds to a two phonon $\beta$ vibration. Our claim, as we justify below, is that the $0_{3}^{+}$state of the full 2D calculations of Fig. 15 is a mixture of a two phonon $\beta$ vibration and a pairing vibration. The bulk structure of the two-phonon $\beta$ vibration [see panel (f) Fig. 17] have four peaks at approximate $q$ values $-140,-90,+100$, and $+220 \mathrm{fm}^{2}$. The bulk structure of the one phonon pairing vibration of panel (b) of Fig. 17, consists in two peaks at -160 and +180 and other two at -50 and $+50 \mathrm{fm}^{2}$. It seems that the most energetically efficient way to combine the node structure of the two $\beta$ phonons and of the one pairing phonon is to favor the two peaks at large deformations (to profit from the large density level) with large pairing correlations and the two at smaller deformation and smaller pairing correlations. That means the two oblate (prolate) peaks at large deformations stemming from both modes merge together and the same happens for the peaks at smaller deformations. The result is the $0_{3}^{+}$state of the full $2 \mathrm{D}$ calculation.

From the above discussion we conclude that the quadrupole degree of freedom provides the bulk structure of the w.f.'s. The 
locations of the minima in the $q$ coordinate being modestly influenced by the pairing degree of freedom. If we look at the $1 \mathrm{D}$ self-consistent path along the $(\delta, \beta)$ plane in Fig. 5(c) we observe little variation of the pairing content of the w.f.'s along this path. As a matter of fact, it has a almost constant value of $\delta \approx 2.5$. This is not the case for the w.f.'s of the full 2D calculations, in particular for the $0_{3}^{+}$state, the candidate to the pairing vibration. What we infer from Figs. 15 and 17 is that the one-phonon pairing vibration mix with the two phonon $\beta$ vibration as to accommodate pieces of the resulting w.f. to different pairing strength.

The conclusion of this part is that the presence of genuine pairing vibration is strongly hindered by the present of minima in the quadrupole degree of freedom which severely modify the node structure of the w.f. The presence of genuine pairing vibration should be limited to double-shell closed nuclei and its closest neighborhood.

\section{PARTICLE-NUMBER DISTRIBUTION IN THE HFB + AMP APPROACH}

An special aspect of the HFB + AMP approach is the particle-number conservation. In this theory the particle number is adjusted, on the average, in the HFB w.f.'s by means of constraints through Lagrange parameters. At the GCM level a correction is performed by the term introduced in Eq. (7). The question we are interested in is this: How good is the particle-number conservation for the states $\left|\Psi^{I, \sigma}\right\rangle$ ? A direct answer to this question is provided by the particle-number distribution of the w.f. $\left|\Psi^{I, \sigma}\right\rangle$. The probability to find an eigenstate of $\hat{N}$ with eigenvalue $N$ in $\left|\Psi^{I, \sigma}\right\rangle$ is given by

$$
\begin{aligned}
W^{N} & =\left|\left\langle N \mid \Psi^{I, \sigma}\right\rangle\right|^{2}=\left\langle\Psi^{I, \sigma} \mid N\right\rangle\left\langle N \mid \Psi^{I, \sigma}\right\rangle \\
& =\left\langle\Psi^{I, \sigma}\left|P^{N}\right| \Psi^{I, \sigma}\right\rangle .
\end{aligned}
$$

In the same way $W^{Z, N}$ represents the probability to have simultaneously an eigenstate of $\hat{Z}$ and $\hat{N}$, with eigenvalues $Z$ and $N$, respectively. Taking into account the definition of $\left|\Psi^{I, \sigma}\right\rangle$ [see Eq. (4)], one obtains

$$
\begin{aligned}
W^{Z, N}= & \left\langle\Psi^{I, \sigma}\left|P^{N} P^{Z}\right| \Psi^{I, \sigma}\right\rangle=\int d q d q^{\prime} d \delta d \delta^{\prime} \\
& \times f^{* b, \sigma}(q, \delta)\left\langle\phi(q, \delta)\left|\hat{P}^{I} \hat{P}^{N} \hat{P}^{Z}\right| \phi\left(q^{\prime}, \delta^{\prime}\right) f^{b, \sigma}\left(q^{\prime}, \delta^{\prime}\right)\right.
\end{aligned}
$$

in terms of known quantities. Because the w.f. $\left|\Psi^{I, \sigma}\right\rangle$ is normalized to unity and $\sum_{N} P^{N}=\sum_{N}|N\rangle\langle N|=1$ is obvious that $\sum_{N, Z} W^{Z, N}=1$.

The usual picture that one has in mind [32] for such distribution is that of a BCS w.f. and only for protons or neutrons. In this case one obtains a Gaussian distribution centered around the value $N$, where $N$ corresponds to the constraint $\langle\mathrm{BCS}|\hat{N}| \mathrm{BCS}\rangle=N$ imposed in the solution of the variational BCS equations. In Fig. 18 we show as an example the distribution $W(Z, N)$ for the nucleus ${ }^{24} \mathrm{Mg}$ and in the 1D case, that means with only $q$ as coordinate, for $I=0^{+}$and for the four lowest eigenstates of the HW equation. In the $X$ axis ( $Y$ axis) we represent the number of protons (neutrons). The color code on the right-hand side of each plot indicates the value of $W(Z, N)$ for each set $(Z, N)$. In this case the
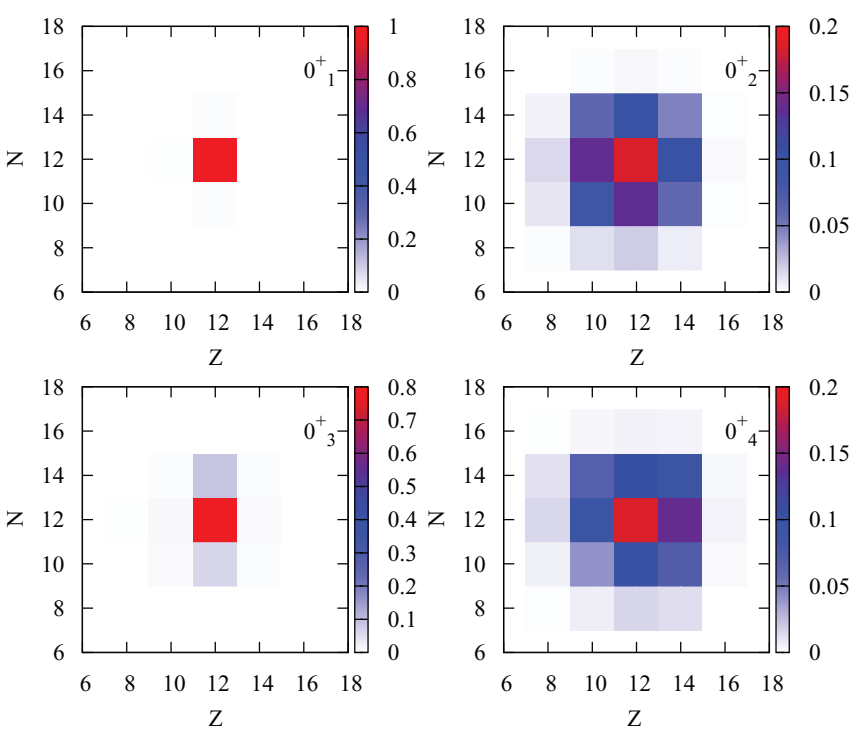

FIG. 18. (Color online) Particle-number distribution in the ground and lowest excited states for ${ }^{24} \mathrm{Mg}$ states in $1 \mathrm{D}$ in the HFB + AMP approach.

ideal distribution would be a 2D Gaussian distribution centered around $Z=N=12$. For the ground state $0_{1}^{+}$we find an almost pure eigenstate of $\hat{Z}$ and $\hat{N}$. A look at the potential well of this nucleus [see panel (c) of Fig. 4] indicates that the w.f. of this state will peak at around $q \approx 60 \mathrm{fm}^{2}$, which corresponds [see panel (d) of Fig. 4] to zero pairing correlations. The distribution of the first excited state $0_{2}^{+}$is shown in the top right panel, where we find a very asymmetric distribution with respect to the line $N+Z=24$. The distribution of the state $0_{3}^{+}$indicates again that we are mainly confronted with zero pairing, whereas the $0_{4}^{+}$distribution is again far from Gaussian. In Fig. 19 we present the same quantity for the two-coordinates $(q, \delta)$ case
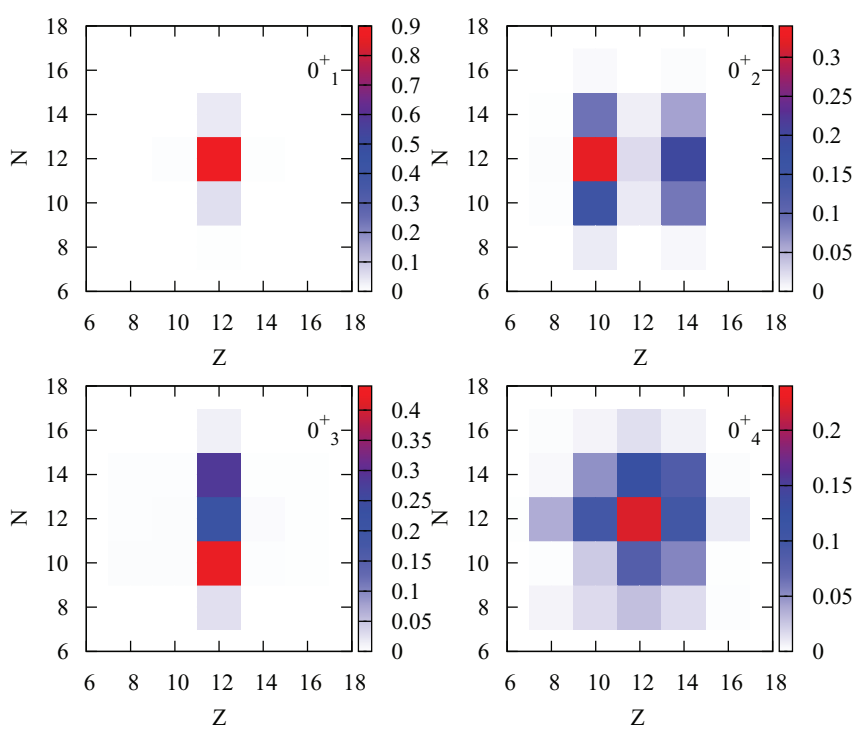

FIG. 19. (Color online) Particle-number distribution in the ground and lowest excited states with $I=0^{+} \hbar$ for ${ }^{24} \mathrm{Mg}$ states in 2D for the HFB + AMP approach. 
and for the same states. The distribution for the $0_{1}^{+}$state is similar to the $1 \mathrm{D}$ case; for the $0_{2}^{+}$we obtain a distribution with a hole at $Z=N=12$ and a very asymmetric distribution. For the $0_{3}^{+}$the maximum is at the wrong number of neutrons and for the $0_{4}^{+}$state though centered around the right value it is not a good Gaussian either. In most of the nuclei the results are similar to these ones.

\section{MISCELLANEOUS CALCULATIONS IN THE PN-VAP + PNAMP APPROACH}

In this section we apply the theory described above to calculate several relevant observables and study the impact of the pairing fluctuations. Because we are mainly interested only in the relevance of the pairing fluctuations we do not discuss thoroughly the physics of the different issues. In the calculations we present results only in the PN-VAP + PNAMP approach.

\section{A. Separation energies}

An interesting quantity is the separation energy. This observable is the difference of two ground-state energies, we do not expect therefore big changes by the explicit consideration of the pairing degree of freedom. In Fig. 20 we display the two neutron separation energies for some magnesium isotopes in the $1 \mathrm{D}$ and 2D calculations as compared with the experimental data. They are defined as the difference between the binding energies:

$$
S_{2 n}(N)=B(Z, N)-B(Z, N-2) .
$$

As expected, the differences between the 1D and 2D are small and both of them show good agreement wit the experimental data.

\section{B. Electric monopole transitions $(E 0)$}

The $E 0$ operator is given by

$$
\hat{T}(E 0)=\sum_{k} e_{k} \hat{r}_{k}^{2} .
$$

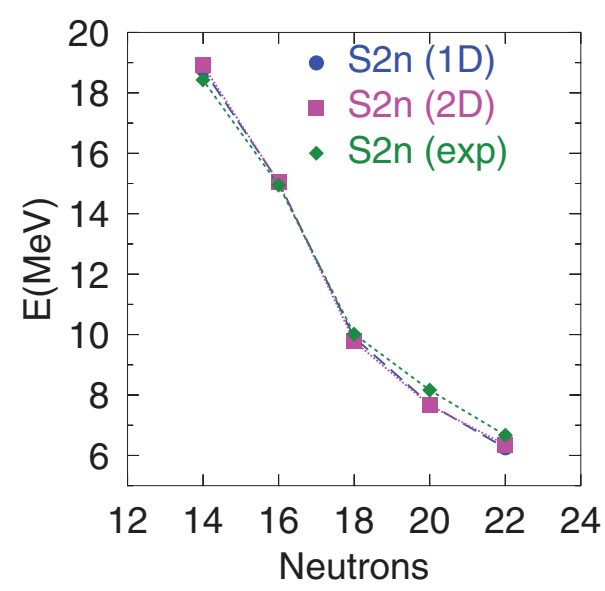

FIG. 20. (Color online) Two neutron separation energies $\left(S_{2 n}\right)$ in $\mathrm{MeV}$ for the magnesium isotopes. The experimental data are taken from Ref. [47].
The diagonal matrix element provides the charge radius and the nondiagonals are related with transitions. Because the radius is strongly related with the shape of the nucleus, the corresponding E0 transitions are also related with the shape of the initial and final states; see Refs. [48,49]. For example, the $\mathrm{EO}\left(\mathrm{O}_{2}^{+} \longrightarrow \mathrm{O}_{1}^{+}\right)$makes it possible to differentiate limiting situations in which two configurations compete for the ground and first excited states. Thus, in the island of inversion the deformed configuration based on two neutrons being excited from the $d_{3 / 2}$ to the intruder orbital $f_{7 / 2}$ keeps pace with the normal spherical one, as illustrated by the well-known case of ${ }^{32} \mathrm{Mg}$, where the intruder state even becomes the ground state [50]. In such a situation of competing configurations and in the absence of mixing one expects either a deformed $0_{1}^{+}$ and a nearly spherical $0_{2}^{+}$state or the other way around. The transition probability is given by

$$
\rho^{2}(E 0)=\frac{1}{R^{4}}\left|\left\langle\Psi_{f}\left|\sum_{k} e_{k} r_{k}^{2}\right| \Psi_{i}\right\rangle\right|^{2},
$$

with $R=1.2 \mathrm{~A}^{1 / 3} ; \Psi_{i}$ and $\Psi_{f}$ are the w.f.'s of the initial and final nuclear states, respectively, in our case the $0_{2}^{+}$and $0_{1}^{+}$ states. As we have seen in the earlier sections the $0^{+}$states are influenced by pairing correlations; thus, we expect some differences between the 1D and 2D calculations.

In Fig. 21 (top panels) we display the excitation energies and the $E 0\left(0_{2}^{+} \longrightarrow 0_{1}^{+}\right)$values for the magnesium isotopes where experimental information is available. Qualitatively, there is not much difference between the $1 \mathrm{D}$ and $2 \mathrm{D}$ predictions for the excitation energy of the $0_{2}^{+}$state, the maximum difference being circa $1 \mathrm{MeV}$. The experimental tendency is fairly well reproduced in both approaches, though the 2D improves somewhat the agreement with the experiment. The discrepant ${ }^{24} \mathrm{Mg}$ value is probably attributable to the fact that this state is a rather pure two-quasiparticle state. In the right top panel the $\rho^{2}\left(E 0,0_{2}^{+} \longrightarrow 0_{1}^{+}\right)$values for the same nuclei are shown. Again there is not a qualitative difference between both predictions. Both display very well the experimental behavior though the 2D calculations predict lower values than the $1 \mathrm{D}$ one. In the particular case of ${ }^{30} \mathrm{Mg}$ the $2 \mathrm{D}$ calculations reduce the $1 \mathrm{D}$ value by a factor of two in such a way that the experimental value is correctly reproduced.

In Table III the charge radius is presented for the magnesium isotopes, in the two approaches and compared with the experiment. Both calculation differ slightly and both show good agreement with the experimental values.

In the middle panels of Fig. 21 we present the excitation energy of the $0_{2}^{+}$states and the monopole transition probability for the $\mathrm{Si}$ isotopes. In these nuclei we observe qualitative and quantitative differences between the 1D and the 2D calculations. Whereas the $1 \mathrm{D}$ predictions provide neither the tendency nor the right value, the 2D calculations improve considerably the agreement with the experimental values. For ${ }^{30} \mathrm{Si}$ the improvement is spectacular, the excitation energy of the $0_{2}^{+}$state is reduced in approximately $1.5 \mathrm{MeV}$ to reproduce the experimental tendency. With respect to the monopole strength, the $2 \mathrm{D}$ is about a factor three smaller than the $1 \mathrm{D}$, getting closer to the experimental value. These large changes have probably to do with the $2 s_{1 / 2}$ subshell closure for $N=16$. 

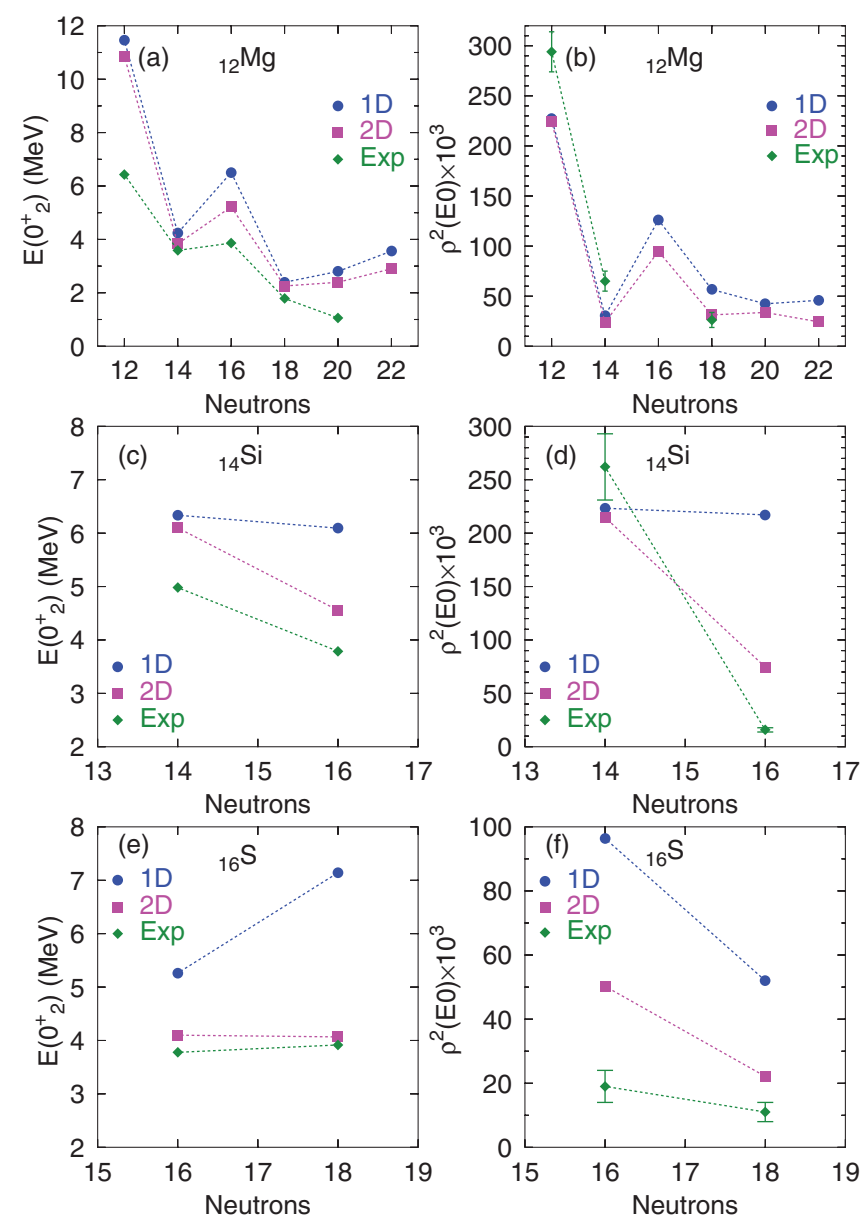

FIG. 21. (Color online) Excitation energies for the $0_{2}^{+}$states and $E 0$ strength $\rho^{2}\left(\mathrm{E} 0,0_{2}^{+} \rightarrow 0_{1}^{+}\right)$for the $\mathrm{Mg}$ isotopes (top panels), the $\mathrm{Si}$ isotopes (middle panels) and $\mathrm{S}$ isotopes (bottom panels). The experimental values are taken from $[47,48,50,51]$.

Finally, in the bottom panels of Fig. 21 we display the results for the sulfur isotopes. Concerning the energy of the $0_{2}^{+}$states we find that as with the $\mathrm{Si}$ isotopes, the $1 \mathrm{D}$ predictions describe very poorly the data. The consideration of the pairing fluctuations again reduce considerably these values as to reproduce very good the experimental values. The same can be said for the monopole strength; the 2D predictions reduce by a factor of two the $1 \mathrm{D}$ calculations in such a way that the data are better reproduced.

TABLE III. Nuclear charge radii for the ground state of the magnesium isotopes. The experimental values are taken from Ref. [52].

\begin{tabular}{lccc}
\hline \hline$\left\langle r^{2}\right\rangle_{\mathrm{ch}}^{1 / 2} \mathrm{fm}^{2}$ & $1 \mathrm{D}$ & $2 \mathrm{D}$ & Exp \\
\hline${ }^{24} \mathrm{Mg}$ & 3.095 & 3.098 & 3.057 \\
${ }^{26} \mathrm{Mg}$ & 3.065 & 3.068 & 3.034 \\
${ }^{28} \mathrm{Mg}$ & 3.078 & 3.082 & 3.070 \\
${ }^{30} \mathrm{Mg}$ & 3.106 & 3.110 & 3.111 \\
${ }^{32} \mathrm{Mg}$ & 3.158 & 3.159 & 3.186 \\
${ }^{34} \mathrm{Mg}$ & 3.210 & 3.213 & \\
\hline \hline
\end{tabular}
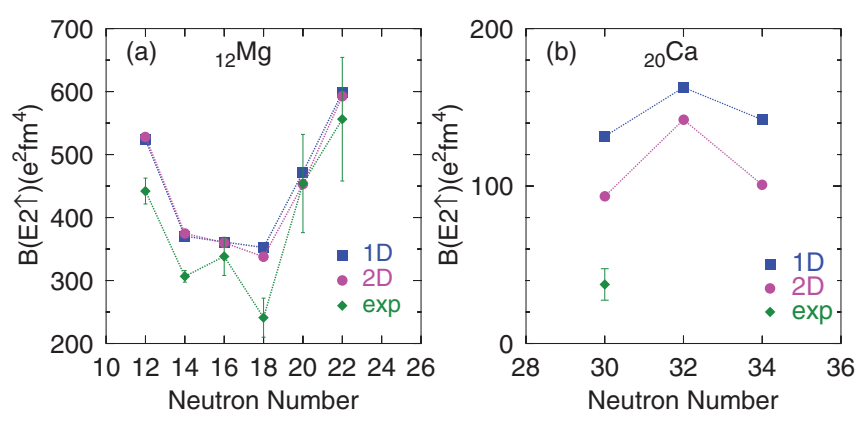

FIG. 22. (Color online) E2 transition probabilities for the $\mathrm{Mg}$ and Ca. Experimental data are taken from Refs. [47,50,53,54].

\section{Quadrupole $E 2\left(0_{1}^{+} \longrightarrow 2_{1}^{+}\right)$transition probabilities}

In Fig. 22 the reduced transition probabilities $B\left(E 2,0_{1}^{+} \longrightarrow 2_{1}^{+}\right)$, for some of the nuclei earlier discussed, are displayed (in the left panel for the magnesium and in the right one for the calcium isotopes). The theoretical predictions in the $1 \mathrm{D}$ approach for the $\mathrm{Mg}$ chain reproduce qualitatively well the experimental behavior despite the fact they are a somewhat larger. The inclusion of the pairing fluctuations, in general, makes the 2D predictions a bit smaller and therefore closer to the experimental data. For the calcium isotopes we find larger contributions of the pairing fluctuations; they amount to a reduction of $30 \%$ of its $1 \mathrm{D}$ values. In the case of the nucleus ${ }^{52} \mathrm{Ti}$ in the $1 \mathrm{D}$ calculations one obtains a $B\left(E 2,0_{1}^{+} \longrightarrow 2_{1}^{+}\right)$of $643.3 e^{2} \mathrm{fm}^{4}$; in the $2 \mathrm{D}$ calculations one obtains a $B\left(E 2,0_{1}^{+} \longrightarrow 2_{1}^{+}\right)$of $601.2 e^{2} \mathrm{fm}^{4}$, compared with the experimental value of $567(51) e^{2} \mathrm{fm}^{4}$. The behavior goes in the same lines as before: tThe pairing fluctuations reduce the, otherwise, too large values, bringing the predictions closer to the experimental values.

\section{CONCLUSIONS}

In this work we have performed a thorough research of the pairing degree of freedom in BMFTs. The quality of the interaction, namely the finite-range density-dependent Gogny forces, guarantees the proper treatment of the pairing correlations.

We have shown that the consideration at the same footing of the pairing degree of freedom and the quadrupole deformation at the different stages of the calculations provides a considerable improvement of the description of many observables of atomic nuclei.

We have underlined the importance of the conservation of symmetries; in particular, we have shown the superiority of the variation after projection for the particle-number case as compared with the plain HFB approach and the projection after variation one. This supremacy manifests itself at the different levels, from the simplest to the most sophisticated ones, in particular, in the solution of the HW equation with the quadrupole and the pairing degrees of freedom as generator coordinates. We have found, in particular, that the absence of PNP leads to a larger linear dependence, implying thereby smaller variational spaces and an unnatural strength concentration. As a consequence the spectra became more compressed than the particle-number projected counterparts. 
The role of the pairing degree of freedom has been analyzed in the most sophisticated approach (VAP-PN + PNAMP) with a large number of observables: spectra, $E 0$ and $E 2$ transition probabilities, separation energies among others. In all studied nuclei we find a better agreement with the experimental data as compared to simpler theories. The pairing vibrations are also thoroughly discussed with the finding that the quadrupole degree of freedom strongly inhibits the presence of genuine pairing vibration.

The validity of our calculations is limited by the absence of explicit single-particle degrees of freedom (two-quasiparticle excitations) as well as the restriction to axial symmetry. The small size of our configuration space also limits the accuracy of our prediction.

In the present work we have analyzed light nuclei mainly in the vicinity of shell closures to keep the configuration space small. The analysis of heavier, spherical, and deformed nuclei will be done for some particular cases in a following paper. We also plan to perform separate constraints on the proton and neutron pairing gap to study specific aspects that are genuine for protons or neutrons.

\section{ACKNOWLEDGMENTS}

The authors acknowledge financial support from the Spanish Ministerio de Ciencia e Innovación under Contract No. FPA2011-29854-C04-04 by the Spanish Consolider-Ingenio 2010 Programme CPAN (CSD2007-00042). N.L.V acknowledges support by the Programa de Formación de Personal Investigador (Ref. No. BES-2010-033107). T.R.R. acknowledges support from BMBF-Verbundforschungsprojekt No. 06DA7047I and Helmholtz International Center for FAIR program.

\section{APPENDIX: DETAILS OF THE DENSITY-DEPENDENT TERM}

In Ref. [7] it was shown that in calculations with a densitydependent interaction and in a particle-number projected approach there are two sources for divergencies. The first one is connected with the neglecting of exchange terms. Obviously, these divergencies can be straightened out by including all missing exchange terms of the interaction. The second one has its origin in the density-dependent term of the interaction which we call $V_{\mathrm{DD}}$. This term was conceived for plain MFAs where only expectation values, i.e., diagonal matrix elements, do appear. Consequently, in the MFA $V_{\mathrm{DD}}$ is constructed to depend on the mean-field density. In theories beyond mean field, for example in PNP, the contribution to the energy of the density-dependent term is given by

$E_{\mathrm{DD}}^{P}=\frac{\left\langle\Phi^{N}\left|\hat{V}_{\mathrm{DD}}[\bar{\rho}(\vec{r})]\right| \Phi^{N}\right\rangle}{\left\langle\Phi^{N} \mid \Phi^{N}\right\rangle}=\frac{\int d \varphi\left\langle\phi\left|\hat{V}_{\mathrm{DD}}[\bar{\rho}(\vec{r})] e^{i \varphi \hat{N}}\right| \phi\right\rangle}{\int d \varphi\left\langle\phi\left|e^{i \varphi \hat{N}}\right| \phi\right\rangle}$,

where $[\bar{\rho}(\vec{r})]$ indicates the explicit dependence of $V_{\mathrm{DD}}$ on a density $\bar{\rho}(\vec{r})$ to be specified. Looking at these expressions it is not obvious which dependence should be used. There are two more or less straightforward prescriptions [55] for $\bar{\rho}(\vec{r})$.

The first prescription is inspired by the following consideration: In the mean-field approximation, the energy is given by $\langle\phi|\hat{H}| \phi\rangle /\langle\phi \mid \phi\rangle$ and $V_{\mathrm{DD}}$ is assumed to depend on the density $\langle\phi|\hat{\rho}| \phi\rangle /\langle\phi \mid \phi\rangle$. However, if the w.f. which describes the nuclear system is the projected w.f. $\left|\Phi^{N}\right\rangle$, we have to calculate the matrix element $\left\langle\Phi^{N}\left|\hat{V}_{\mathrm{DD}}\right| \Phi^{N}\right\rangle /\left\langle\Phi^{N} \mid \Phi^{N}\right\rangle$ [see the middle term in Eq. (A1)]. It seems reasonable, therefore, to use in $V_{\mathrm{DD}}$ the density $\bar{\rho}(\vec{r}) \equiv \rho^{N}(\vec{r})=\left\langle\Phi^{N}|\hat{\rho}| \Phi^{N}\right\rangle /\left\langle\Phi^{N} \mid \Phi^{N}\right\rangle$, i.e., the projected density. One has to be aware that this prescription can be used only in the case of the PNP, where one projects in the gauge space associated with the particlenumber operator and which has nothing to do with the spacial coordinates. In the case of symmetries associated with $\vec{r}$ like the angular-momentum or parity projection, one has to work with the second prescription.

The second prescription has been guided by the choice usually done in the GCM with density-dependent forces [33]. The philosophy behind this prescription is the following: To evaluate Eq. (A1) we have to calculate matrix elements between different product w.f.'s $|\phi\rangle$ and $|\tilde{\phi}\rangle\left(|\tilde{\phi}\rangle=e^{i \varphi \hat{N}}|\phi\rangle\right)$ [see last term in Eq. (A1)]. Then, to calculate matrix elements of the form $\left\langle\phi\left|\hat{V}_{\mathrm{DD}}\right| \tilde{\phi}\right\rangle\langle\phi \mid \tilde{\phi}\rangle$ we choose the mixed density
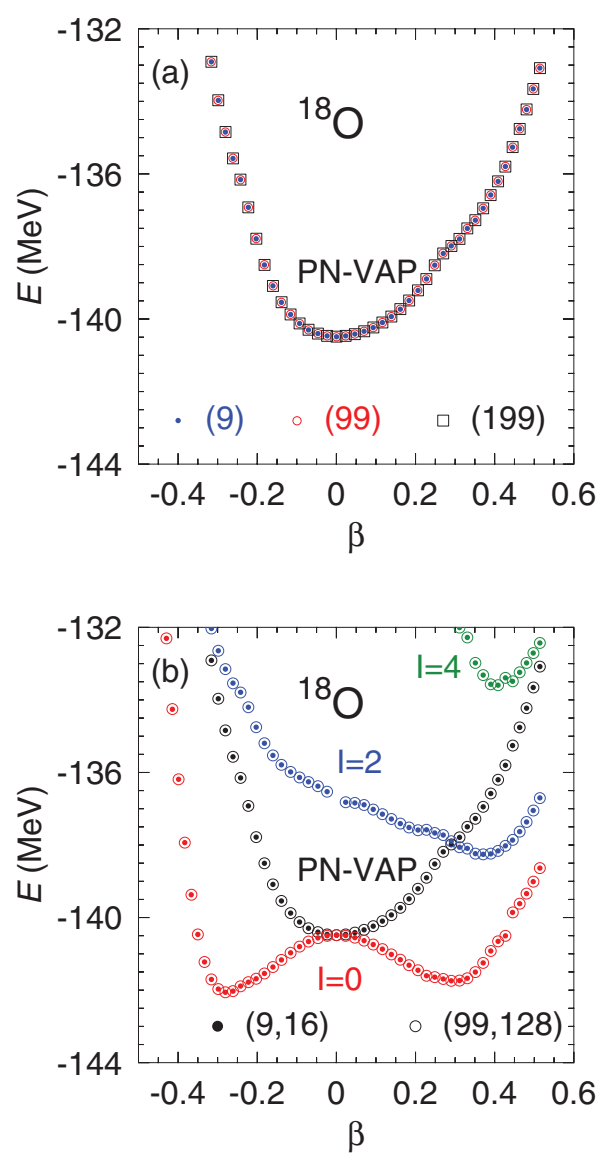

FIG. 23. (Color online) (a) Potential energy surfaces in the PNVAP approach for different number of integration points. (b) Potential energy surfaces in the PN-VAP approach and in the PNAMP approach for $I=0,2$, and 4 for different number of integration points. 
$\bar{\rho}(\vec{r})=\rho_{\varphi}(\vec{r})=\langle\phi|\hat{\rho}(\vec{r})| \tilde{\phi}\rangle\langle\phi \mid \tilde{\phi}\rangle$ to be used in $\hat{V}_{\mathrm{DD}}$. This approach is called the mixed density prescription.

Both prescriptions have been tested with the Gogny force in the Lipkin Nogami approach [56] and practically no difference was found in the numerical applications. One should notice that in the second prescription $\bar{\rho}(\vec{r})$ depends on the angle $\varphi$ at variance with the first prescription.

It has been shown in Ref. [7] for the PNP that the projected prescription is free from divergences while the mixed prescription may present some problems.

To get rid of the divergencies in our calculations we include all exchange terms of the Gogny force. Concerning the densitydependent term we use the projected density in the PNP case and the mixed density prescription in the AMP and in the GCM cases.

To illustrate the absence of divergences under these conditions we discuss the paradigmatic case of ${ }^{18} \mathrm{O}$ used by Bender and collaborators [35] to show the presence of divergencies in the case of the Skyrme force and without taking care to remedy the above-mentioned problems. In Fig. 1 of Ref. [35] the potential energy of the nucleus ${ }^{18} \mathrm{O}$ was plotted against the $\beta$ degree of freedom taking 5 and 199 integration points in the discretization of the projection of the number of particles. In this figure one can observe two poles at $\beta \approx 0.22$ and $\beta \approx-0.3$ for 199 points. We now present in Fig. 23(a) the same plot for the Gogny force calculated in the way mentioned above, taking 9, 99, and 199 integration points. One can immediately observe not only the absence of any divergency but also the perfect convergence of the calculations.

Concerning the AMP the current status is that the studies on PNP $[35,36]$ have been simply extrapolated to this case. However, to our knowledge, an explicit study of the existence of divergences and/or steps by the use of the mixed density prescription in the AMP case has not been carried out. Even more, we have looked explicitly for such an ill behavior in many calculations with axial and triaxial AMP and we have never found any hint of them. As an example, in Fig. 23(b) we also show the results for simultaneous projection of particle number and angular momentum for $I=0 \hbar, 2 \hbar$, and $4 \hbar$. We present two calculations, one with 9 (16) integration points for particle number (angular momentum) and another with 99 (128), respectively. Again, in contrast to Refs. [35,36], the absence of divergences/steps and the good convergence are manifest.

The conclusion with respect to the use of the mixed-density prescription in the case of the AMP with the Gogny interaction is that either there are no problems with its nonanalicity in the complex plane or they appear, contrary to the PNP case, so seldom that the probability of finding them in practical calculations is quite negligible. Two last concluding remarks: First, obviously a detailed studied should be performed to clarify this issue; second, our conclusion is limited to the present type of calculations, i.e., without time-reversal breaking.
[1] M. Bender, P.-H. Heenen, and P.-G. Reinhard, Rev. Mod. Phys. 75, 121 (2003).

[2] H. Mang, Phys. Rep. 18, 325 (1975).

[3] J. L. Egido and P. Ring, Nucl. Phys. A 383, 189 (1982).

[4] K. Schmid, Prog. Part. Nucl. Phys. 52, 565 (2004).

[5] K. Dietrich, H. J. Mang, and J. H. Pradal, Phys. Rev. 135, B22 (1964).

[6] J. L. Egido and P. Ring, Nucl. Phys. A 388, 19 (1982).

[7] M. Anguiano, J. L. Egido, and L. M. Robledo, Nucl. Phys. A 696, 467 (2001).

[8] M. Bender and P.-H. Heenen, Phys. Rev. C 78, 024309 (2008).

[9] J. M. Yao, J. Meng, P. Ring, and D. Vretenar, Phys. Rev. C 81, 044311 (2010).

[10] R. Rodríguez-Guzman, J. L. Egido, and L. M. Robledo, Nucl. Phys. A 709, 201 (2002).

[11] T. R. Rodríguez and J. L. Egido, Phys. Rev. C 81, 064323 (2010).

[12] R. Broglia and V. Zelevinsky (eds.), Fifty Years of Nuclear BCS (World Scientific, Singapore, 2013).

[13] M. Baranger, Phys. Rev. 122, 992 (1961).

[14] C. Siegal and R. Sorensen, Nucl. Phys. A 184, 81 (1972).

[15] Y. R. Shimizu, J. D. Garrett, R. A. Broglia, M. Gallardo, and E. Vigezzi, Rev. Mod. Phys. 61, 131 (1989).

[16] D. Bés, R. Broglia, J. Dudek, W. Nazarewicz, and Z. Szymański, Ann. Phys. 182, 237 (1988).

[17] M. Kyotoku and H.-T. Chen, Phys. Rev. C 36, 1144 (1987).

[18] A. Staszczak, S. Piłat, and K. Pomorski, Nucl. Phys. A 504, 589 (1989).

[19] G. Potel, F. Barranco, F. Marini, A. Idini, E. Vigezzi, and R. A. Broglia, Phys. Rev. Lett. 107, 092501 (2011).
[20] N. Pillet, V. G. Zelevinsky, M. Dupuis, J.-F. Berger, and J. M. Daugas, Phys. Rev. C 85, 044315 (2012).

[21] R. Broglia, O. Hansen, and C. Riedel, Adv. Nucl. Phys. 6, 287 (1973).

[22] D. Bes, R. Broglia, R. Perazzo, and K. Kumar, Nucl. Phys. A 143, 1 (1967).

[23] A. Góźdź, K. Pomorski, M. Brack, and E. Werner, Nucl. Phys. A 442, 26 (1985).

[24] L. Pròchniak, K. Zając, K. Pomorski, S. Rohoziński, and J. Srebrny, Nucl. Phys. A 648, 181 (1999).

[25] K. Zając, L. Pròchniak, K. Pomorski, S. Rohoziński, and J. Srebrny, Nucl. Phys. A 653, 71 (1999).

[26] M. A. Fernández and J. L. Egido, Phys. Rev. B 68, 184505 (2003)

[27] M. A. Fernández and J. L. Egido, Eur. Phys. J B 48, 305 (2005).

[28] A. Faessler, F. Grümmer, A. Plastino, and F. Krmpotić, Nucl. Phys. A 217, 420 (1973).

[29] J. Meyer, P. Bonche, J. Dobaczewski, H. Flocard, and P. H. Heenen, Nucl. Phys. A 533, 307 (1991).

[30] N. L. Vaquero, T. R. Rodríguez, and J. L. Egido, Phys. Lett. B 704, 520 (2011).

[31] J. L. Egido, J. Lessing, V. Martin, and L. M. Robledo, Nucl. Phys. A 594, 70 (1995).

[32] P. Ring and P. Schuck, The Nuclear Many Body Problem (Springer, Berlin, 1980).

[33] P. Bonche, J. Dobaczewski, H. Flocard, P. H. Heenen, and J. Meyer, Nucl. Phys. A 510, 466 (1990).

[34] J. F. Berger, M. Girod, and D. Gogny, Nucl. Phys. A 428, 23 (1984). 
[35] M. Bender, T. Duguet, and D. Lacroix, Phys. Rev. C 79, 044319 (2009).

[36] J. Dobaczewski, M. V. Stoitsov, W. Nazarewicz, and P.-G. Reinhard, Phys. Rev. C 76, 054315 (2007).

[37] G. Hupin, D. Lacroix, and M. Bender, Phys. Rev. C 84, 014309 (2011).

[38] J. Egido, L. Robledo, and Y. Sun, Nucl. Phys. A 560, 253 (1993).

[39] M. Anguiano, J. L. Egido, and L. M. Robledo, Nucl. Phys. A 683, 227 (2001).

[40] A. Kamlah, Z. Phys. 216, 52 (1968).

[41] H. Zduńczuk, J. Dobaczewski, and W. Satuła, Int. J. Mod. Phys. E 16, 377 (2007).

[42] T. R. Rodríguez, J. L. Egido, L. M. Robledo, and R. RodríguezGuzmán, Phys. Rev. C 71, 044313 (2005).

[43] T. Otsuka, R. Fujimoto, Y. Utsuno, B. A. Brown, M. Honma, and T. Mizusaki, Phys. Rev. Lett. 87, 082502 (2001).

[44] J. I. Prisciandaro, P. F. Mantica, B. A. Brown, D. W. Anthony, M. W. Cooper, A. Garcia, D. E. Groh, A. Komives, W. Kumarasiri, P. A. Lofy et al., Phys. Lett. B 510, 17 (2001).

[45] T. R. Rodríguez and J. L. Egido, Phys. Rev. Lett. 99, 062501 (2007).

[46] D. Steppenbeck, S. Takeuchi, N. Aoi, P. Doornenbal, M. Matsushita, H. Wang, H. Baba, N. Fukuda, S. Go, M. Honma et al., Nature (London) 502, 207 (2013).
[47] Endsf database, http://www.nndc.bnl.gov/endsf.

[48] J. Wood, E. Zganjar, C. D. Coster, and K. Heyde, Nucl. Phys. A 651, 323 (1999).

[49] K. Heyde and J. L. Wood, Rev. Mod. Phys. 83, 1467 (2011).

[50] W. Schwerdtfeger, P. G. Thirolf, K. Wimmer, D. Habs, H. Mach, T. R. Rodriguez, V. Bildstein, J. L. Egido, L. M. Fraile, R. Gernhäuser et al., Phys. Rev. Lett. 103, 012501 (2009).

[51] T. Kibédi and R. Spear, At. Data Nucl. Data Tables 89, 77 (2005).

[52] D. T. Yordanov, M. L. Bissell, K. Blaum, M. De Rydt, C. Geppert, M. Kowalska, J. Krämer, K. Kreim, A. Krieger, P. Lievens et al., Phys. Rev. Lett. 108, 042504 (2012).

[53] D.-C. Dinca, R. V. F. Janssens, A. Gade, D. Bazin, R. Broda, B. A. Brown, C. M. Campbell, M. P. Carpenter, P. Chowdhury, J. M. Cook et al., Phys. Rev. C 71, 041302 (2005).

[54] J. J. Valiente-Dobón, D. Mengoni, A. Gadea, E. Farnea, S. M. Lenzi, S. Lunardi, A. Dewald, T. Pissulla, S. Szilner, R. Broda et al., Phys. Rev. Lett. 102, 242502 (2009).

[55] A. Valor, J. Egido, and L. Robledo, Nucl. Phys. A 665, 46 (2000)

[56] A. Valor, J. Egido, and L. Robledo, Phys. Lett. B 392, 249 (1997). 